NASA Technical Memorandum 106838

\title{
Driving Force Analysis in an Infinite Anisotropic Plate With Multiple Crack Interactions
}

Wieslaw K. Binienda

The University of Akron Akron, Ohio

and

Steven M. Arnold Lewis Research Center Cleveland, Ohio

January 1995

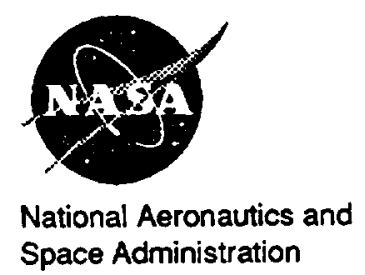

(NASA-TM-106838) DRIVING FORCE

N95-19782

ANALYSIS IN AN INFINITE ANISOTROPIC PLATE WITH MULTIPLE CRACK

INTERACTIONS (NASA. LEWIS Research Center) $40 \mathrm{P}$ 


\title{
Driving Force Analysis in an Infinite Anisotropic Plate With Multiple Crack Interactions
}

\author{
Wieslaw K. Binienda \\ Department of Civil Engineering \\ The University of Akron \\ Akron, $\mathrm{OH} 44325-3905$
}

\author{
Steven M. Arnold \\ NASA Lewis Research Center \\ Cleveland, $\mathrm{OH} 44135$
}

\begin{abstract}
The methodology and a rigorous solution formulation are presented for stress intensity factors (SIF's, $k$ ) and total strain energy release rates (SERR, $G_{T}$ ) of a multicracked plate, that has fully interacting cracks and is subjected to a far-field arbitrary stress state. The fundamental perturbation problem is derived, and the steps needed to formulate the system of singular integral equations whose solution gives rise to the evaluation of the SIF's are identified. Parametric studies are conducted for two, three and four crack problems. The sensitivity and characteristics of the model is demonstrated.
\end{abstract}

\section{Nomenclature}

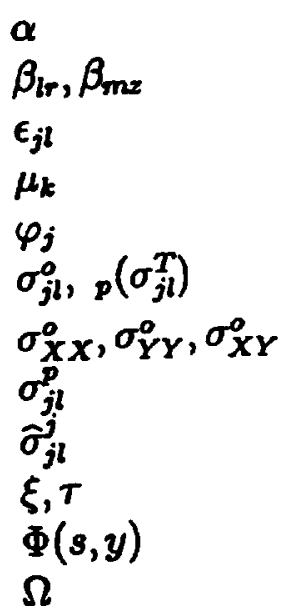

- inclination angle between inner tips of two parallel cracks

- direction cosines between two local coordinate systems

- strain tensor

- four roots of the characteristic equation

- angle defining orientation of local coordinate system

- far-field and total stress field, respectively

- components of stress in global coordinate system

- stress from the perturbation problem in $p^{\text {th }}$ local frame

- transformed $j^{\text {th }}$ crack stressed to the $p^{\text {th }}$ local frame

- normalized real variables

- Fourier transform of the stress function with respect to $x$

- angle between $L-T$ and $X-Y$ coordinate systems 


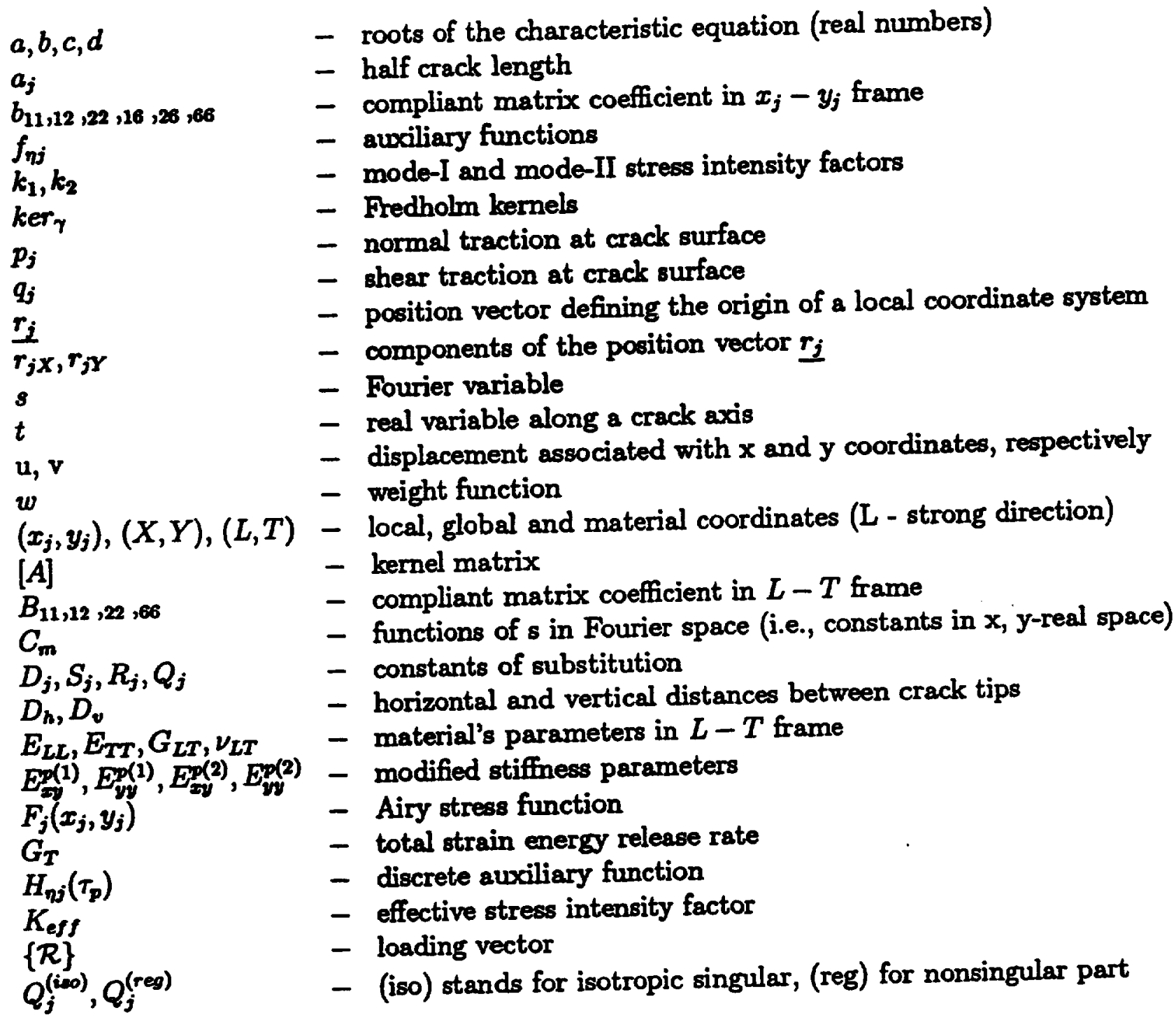

\section{INTRODUCTION}

Consider multiple cracks embedded in an infinite anisotropic plate (Fig. 1(a)). The plate is under a far-field stress denoted by $\sigma_{j l}^{\circ}$, (in particular $\sigma_{X X}^{\circ}, \sigma_{Y Y}^{\circ}$, and $\sigma_{X Y}^{\circ}$, where $(\mathrm{X}, \mathrm{Y})$ is the global coordinate system), and the cracks are defined in their local frames $\left(x_{j}, y_{j}\right)$ (Fig. 1(b)). The origin of each local frame is defined by the position vector $r_{j}$, and the orientation of the local frame with respect to the global frame is defined by the angle $\varphi_{j}$. Each crack is symmetrically situated within its own coordinate system and is $2 a_{j}$ long, as shown in Fig. 1(b).

The general solution formulation can be outlined in four basic steps. The first step is to derive the local stress equations for each crack in its respective local coordinate system. This derivation is achieved by defining the fundamental problem; that is a single crack in an infinite anisotropic plane (Fig. 1(b)). The fundamental problem is then decomposed into two subproblems: the problem of the undamaged plate containing an imaginary crack (Fig. 1(c)), and the perturbation problem (Fig. 1(d)) of a plate with a single crack subjected to the appropriate crack-surface tractions which are found from the solution of the complementary undamaged problem. The analysis of the perturbation 
problem leads to singular stresses that govern local crack-tip behavior.

The second step is to formulate the total perturbation stress field for each crack, which includes the interaction of all cracks through the summation of the transformed local stresses of all other cracks. In the third step of the formulation, the total stress equations are normalized. A set of Cauchy-type singular integral equations, expressed in terms of unknown auxiliary functions, is obtained by subjecting the total perturbation stress equations to the crack-surface traction field at each crack location. The fourth and final step of the formulation is to express the stress intensity factors (SIF's, $k_{1}$ and $k_{2}$ ) in terms of the discrete awdiliary functions $H_{\eta j}\left(\tau_{p}\right)$ evaluated at each crack tip. These discrete auxiliary functions are obtained by implementing of the Lobatto-Chebyshev collocation technique. Finally, the strain energy release rate (SERR, $G_{T}$ ) is calculated in terms of SIF's.

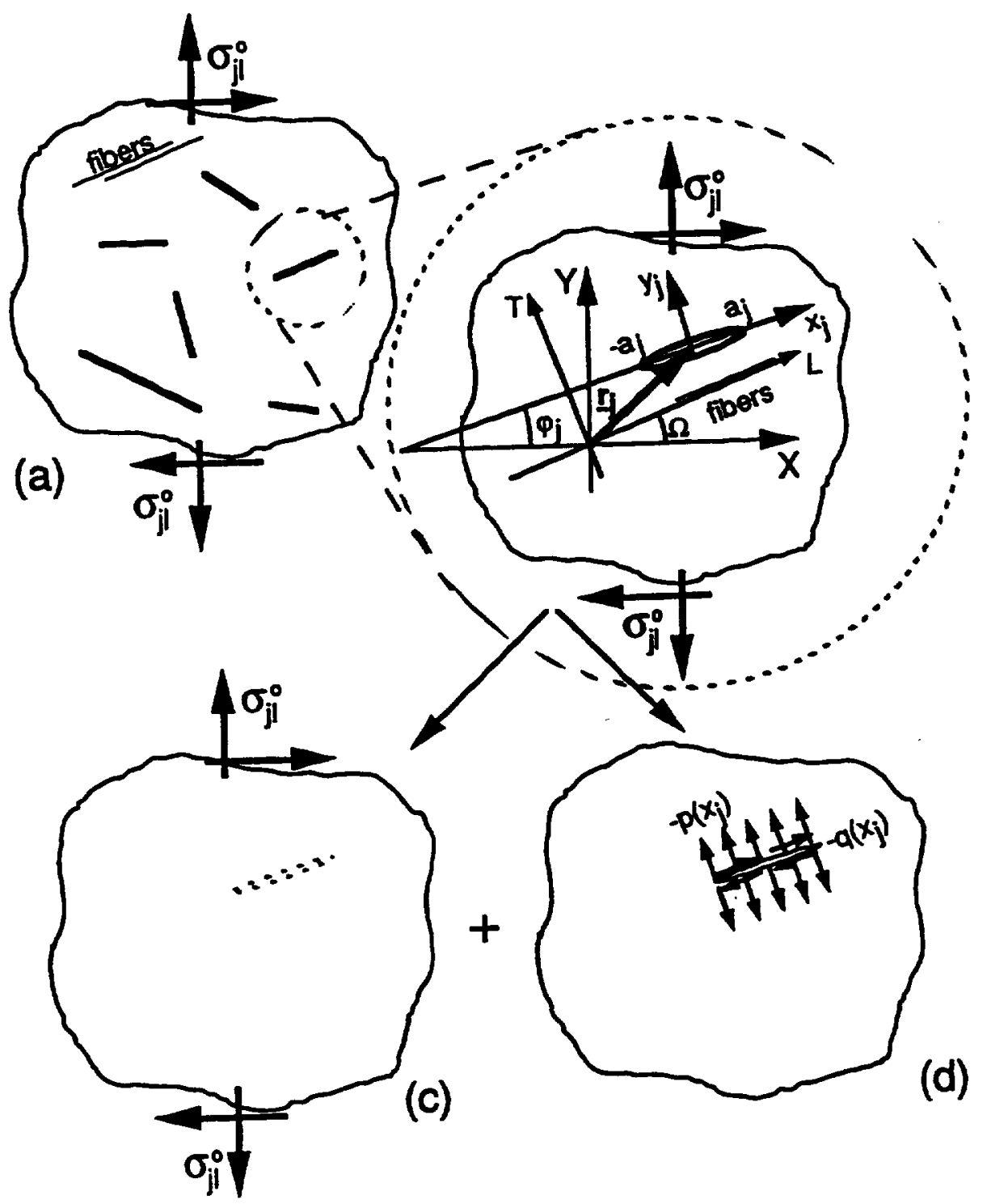

(b)

Fundamental problem for $j$ th crack. (c) Undamaged plate. (d) Perturbation problem. 


\section{LOCAL STRESS FORMULATION}

Consider the fundamental problem (Fig. 1(b)), which is defined as a single crack in an infinite anisotropic plate; its solution can be obtained by decomposing it into an undamaged problem (Fig. 1(c)) and a perturbation problem (Fig. 1(d)). The essence of this decomposition is that the traction forces applied along the crack surface in the perturbation problem are the opposite of the obtained stress field of the undamaged plate at the particular location of the imaginary crack. As a result, the undamaged plate's traction field can be defined in terms of the normal $\left(p_{j}\right)$ and shear $\left(q_{j}\right)$ stress components along the imaginary crack surface:

$$
\begin{aligned}
& p_{j}\left(x_{j}\right)=\sigma_{y_{j} y_{j}}\left(x_{j}, 0\right) \\
& q_{j}\left(x_{j}\right)=\sigma_{x_{j} y_{j}}\left(x_{j}, 0\right)
\end{aligned}
$$

where

$$
\begin{gathered}
\sigma_{y_{j} y_{j}}\left(x_{j}, 0\right)=\sigma_{X X}^{\circ} \sin ^{2} \varphi_{j}+\sigma_{Y Y}^{\circ} \cos ^{2} \varphi_{j}-\sigma_{X Y}^{\circ} \sin 2 \varphi_{j} \\
\sigma_{x_{j} y_{j}}\left(x_{j}, 0\right)=-\frac{\sigma_{X X}^{\circ}-\sigma_{Y Y}^{\circ} \sin 2 \varphi_{j}+\sigma_{X Y}^{\circ} \cos 2 \varphi_{j}}{2} \sin
\end{gathered}
$$

The mixed boundary conditions for the perturbation part of the fundamental problem (Fig. 1(d)) are expressed in terms of stresses

$$
\sigma_{y_{j} y_{j}}=-p_{j}\left(x_{j}\right) \quad \text { and } \quad \sigma_{x_{j} y_{j}}=-q_{j}\left(x_{j}\right)
$$

along the crack surface (i.e., $y_{j}=0$ and $-a_{j} \leq x_{j} \leq a_{j}$ ), and in terms of continuity of displacements

$$
v^{+}=v^{-} \quad \text { and } u^{+}=u^{-}
$$

outside of the crack (i.e., $y_{j}=0$ and $\left|x_{j}\right|>a_{j}$, see Fig. 1(b)). Here the superscript " $+^{n}$ indicates the value of displacement at a point approached from the positive side of the plate, (i.e., $y>0$ ), whereas " - indicates the same point approached from the negative side of the plate (i.e., $y<0)$.

The governing equation for the preceding two-dimensional anisotropic plate problem can be expressed in terms of the Airy stress function $F_{j}\left(x_{j}, y_{j}\right)$ as

$$
\frac{\partial^{4} F}{\partial x^{4}}+\gamma_{1} \frac{\partial^{4} F}{\partial x^{3} \partial y}+\gamma_{2} \frac{\partial^{4} F}{\partial x^{2} \partial y^{2}}+\gamma_{3} \frac{\partial^{4} F}{\partial x \partial y^{3}}+\gamma_{4} \frac{\partial^{4} F}{\partial y^{4}}=0
$$

where

$$
\gamma_{1}=-\frac{2 b_{25}}{b_{22}} ; \quad \gamma_{2}=\frac{2 b_{12}+b_{35}}{b_{22}} ; \quad \gamma_{3}=-\frac{2 b_{18}}{b_{22}} ; \quad \text { and } \quad \gamma_{4}=\frac{b_{11}}{b_{22}}
$$

Here, for general two-dimensional anisotropy $b_{11}, b_{22}, b_{12}, b_{16}, b_{26}$, and $b_{66}$ are independent.

In this paper, we will be primarily concerned with applications involving unidirectional fiber reinforced composites; these can be idealized at the macrolevel as a pseudohomogeneous transversely isotropic material. Consequently, the preceding six independent constants now become dependent on the four independent elastic constants, $E_{L L}, E_{T T}, G_{L T}$ and $\nu_{L T}$, where $(\mathrm{L}, \mathrm{T})$ is the material coordinate system rotated by the angle $\Omega$ with respect to $(\mathrm{X}, \mathrm{Y})$, see Fig. $1(\mathrm{~b})$. 
Thus,

$$
\begin{aligned}
& b_{11}=B_{11} \cos ^{4}(\Omega-\varphi)+\left(2 B_{12}+B_{66}\right) \sin ^{2}(\Omega-\varphi) \cos ^{2}(\Omega-\varphi)+B_{22} \sin ^{4}(\Omega-\varphi) \\
& b_{22}=B_{22} \cos ^{4}(\Omega-\varphi)+\left(2 B_{12}+B_{66}\right) \sin ^{2}(\Omega-\varphi) \cos ^{2}(\Omega-\varphi)+B_{11} \sin ^{4}(\Omega-\varphi) \\
& b_{12}=B_{12}+\left(B_{11}+B_{22}-2 B_{12}-B_{66}\right) \sin ^{2}(\Omega-\varphi) \cos ^{2}(\Omega-\varphi) \\
& b_{66}=B_{66}+\left(B_{11}+B_{22}-2 B_{12}-B_{66}\right) \sin ^{2}(\Omega-\varphi) \cos ^{2}(\Omega-\varphi) \\
& b_{16}=\left[B_{22} \sin ^{2}(\Omega-\varphi)-B_{11} \cos ^{2}(\Omega-\varphi)+\frac{1}{2}\left(2 B_{12}+B_{66}\right) \cos 2(\Omega-\varphi)\right] \sin 2(\Omega-\varphi) \\
& b_{26}=\left[\cos ^{2}(\Omega-\varphi)-\sin ^{2}(\Omega-\varphi)-\frac{1}{2}\left(2 B_{12}+B_{66}\right) \cos 2(\Omega-\varphi)\right] \sin 2(\Omega-\varphi)
\end{aligned}
$$

where

$$
B_{11}=\frac{1}{E_{L L}} ; \quad B_{22}=\frac{1}{E_{T T}} ; \quad B_{12}=\frac{-\nu_{I T T}}{E_{L L}} ; \quad \text { and } \quad B_{66}=\frac{1}{G_{L T}}
$$

Note that this special case of transverse isotropy does not diminish from the generality of the subsequent solution for the general anisotropic case; all that is required to recover the general solution is to experimentally identify the six independent constants used in equation (9).

A rigorous solution for this stress function can be obtained by employing the Fourier transform. Assume the stress function to be expressed as

$$
F(x, y)=\frac{1}{2 \pi} \int_{-\infty}^{\infty} \sum_{m=1}^{4} C_{m} e^{\mu_{m} y s} e^{-i \infty x} d s
$$

Then, on substitution into eq. (7) the characteristic equation is obtained. It has four complex roots, which take the following form:

$$
\begin{gathered}
\mu_{1}=a+i b ; \quad \mu_{2}=c+i d \\
\mu_{3}=-a+i b ; \quad \mu_{4}=-c+i d
\end{gathered}
$$

where $a$ and $c>0$.

The Airy stress function must also satisfy the physical requirement that the stress function is finite throughout the domain of the plate. Therefore, the following forms of $F_{j}\left(x_{j}, y_{j}\right)$, which are automatically bounded at infinity, can be used for the upper half plane (for $y>0$ ),

$$
F\left(x, y^{+}\right)=\frac{1}{2 \pi} \int_{-\infty}^{\infty}\left[C_{1} e^{(i b s-a|s|) y}+C_{2} e^{(i d s-c|s|) y}\right] e^{-i a x} d s
$$

and for the lower half plane (for $y<0$ ),

$$
F\left(x, y^{-}\right)=\frac{1}{2 \pi} \int_{-\infty}^{\infty}\left[C_{3} e^{(i b s+a|s|) v}+C_{4} e^{(i d s+c|a|) y}\right] e^{-i s x} d s
$$

Note: Constants $C_{m}$ for $\mathrm{j}=1,2,3$, and 4 are functions of the Fourier variable $s$ and are determined by using the local stress continuity conditions at the boundaries between the half planes $(y=0)$ and by using the perturbation boundary conditions subsequent to the determination of the total stresses at each crack location.

The stresses within the upper and lower half planes are calculated by using the second derivatives of the stress functions [1]. Therefore, the stresses for the upper half plane are

$$
\sigma_{x x}^{(+)}=\frac{1}{2 \pi} \int_{-\infty}^{\infty}\left[C_{1}(-a|s|+i b s)^{2} e^{(i b s-a \mid s) y}+C_{2}(-c|s|+i d s)^{2} e^{(i d s-c|s|) y}\right] e^{-i s x} d s
$$




$$
\begin{gathered}
\sigma_{y y}^{(+)}=-\frac{1}{2 \pi} \int_{-\infty}^{\infty} s^{2}\left[C_{1} e^{(i b s-a|s|) y}+C_{2} e^{(i d s-c|s|) y}\right] e^{-i s x} d s \\
\sigma_{x y}^{(+)}=\frac{1}{2 \pi} \int_{-\infty}^{\infty} i s\left[C_{1}(-a|s|+i b s) e^{(i b s-a|s|) y}+C_{2}(-c|s|+i d s) e^{(i d s-c|s|) y}\right] e^{-i s x} d s
\end{gathered}
$$

and for the lower half plane

$$
\begin{gathered}
\sigma_{x x}^{(-)}=\frac{1}{2 \pi} \int_{-\infty}^{\infty}\left[C_{3}(a|s|+i b s)^{2} e^{(i b s+a|d|) y}+C_{4}(c|s|+i d s)^{2} e^{(i d s+c|a|) y}\right] e^{-i s x} d s \\
\sigma_{y y}^{(-)}=-\frac{1}{2 \pi} \int_{-\infty}^{\infty} s^{2}\left[C_{3} e^{(i b s+a|s|) y}+C_{4} e^{(i d s+c|s|) y}\right] e^{-i s x} d s \\
\sigma_{x y}^{(-)}=\frac{1}{2 \pi} \int_{-\infty}^{\infty} i s\left[C_{3}(a|s|+i b s) e^{(i b s+a \mid a) y}+C_{4}(c|s|+i d s) e^{(i d s+c|a|) y}\right] e^{-i s x} d s
\end{gathered}
$$

The continuity conditions for local stresses $\sigma_{y y}$ and $\sigma_{x y}$ are identically satisfied, given

$$
C_{1}+C_{2}=C_{3}+C_{4}
$$

and

$$
C_{1}(b s+i a|s|)+C_{2}(d s+i c|s|)=C_{3}(b s-i a|s|)+C_{4}(d s-i c|s|)
$$

respectively.

The solution of equations (20) and (21) for $C_{3}$ and $C_{4}$ in terms of $C_{1}$ and $C_{2}$ can be written in the following form:

$$
\begin{aligned}
& C_{3}=S_{1} C_{1}+S_{2} C_{2} \\
& C_{4}=S_{3} C_{1}+S_{4} C_{2}
\end{aligned}
$$

where

$$
\begin{aligned}
S_{1} & =\frac{|s|(a+c)+i(d-b) s}{|s|(c-a)+i(d-b) s} \\
S_{2} & =\frac{2 c|s|}{|s|(c-a)+i(d-b) s} \\
S_{3} & =-\frac{2 a|s|}{|s|(c-a)+i(d-b) s} \\
S_{4} & =-\frac{|s|(a+c)-i(d-b) s}{|s|(c-a)+i(d-b) s}
\end{aligned}
$$

The strains are calculated by using the generalized Hooke's law. The normal strains are

$$
\begin{aligned}
& \varepsilon_{x x}=b_{11} \sigma_{x x}+b_{12} \sigma_{y y}+b_{16} \sigma_{x y} \\
& \varepsilon_{y y}=b_{12} \sigma_{x x}+b_{22} \sigma_{y y}+b_{26} \sigma_{x y}
\end{aligned}
$$

From eqs. (24) the strains for the upper and lower half planes can be obtained. Then by using the strain-displacement relations [1]; the displacements for the upper and lower half plane can be obtained:

$$
\begin{aligned}
& u(x, y)=\int \varepsilon_{x x} d x \\
& v(x, y)=\int \varepsilon_{y y} d y
\end{aligned}
$$

To obtain the singular integral equations we introduce the following auxiliary functions: 


$$
\begin{aligned}
& f_{1}(x)=\frac{\partial}{\partial x}\left[u^{+}(x, 0)-u^{-}(x, 0)\right] \\
& f_{2}(x)=\frac{\partial}{\partial x}\left[v^{+}(x, 0)-v^{-}(x, 0)\right]
\end{aligned}
$$

Expressions for the unknown constants $C_{1}$ and $C_{2}$ can be determined in terms of these auxiliary functions, since we know that $f_{1}(t)$ and $f_{2}(t)$ are nonzero only within the crack region (i.e., $-a<t<a$ ). Therefore,

$$
\begin{gathered}
C_{1}=\int_{-a}^{a} \frac{D_{4} f_{1}(t)-D_{2} f_{2}(t)}{D_{1} D_{4}-D_{2} D_{3}} e^{i s t} d t \\
C_{2}=-\int_{-a}^{a} \frac{D_{3} f_{1}(t)-D_{1} f_{2}(t)}{D_{1} D_{4}-D_{2} D_{3}} e^{i s t} d t
\end{gathered}
$$

where

$$
\begin{aligned}
& D_{1}=2 a|s|[|s|(a+c)+i(d-b) s] b_{11} \\
& D_{2}=2 c|s|[|s|(a+c)-i(d-b) s] b_{11} \\
& D_{3}=2 a \frac{|s| s[s(d-b)-i(a+c)|s|]}{\left(a^{2}+b^{2}\right)(c|s|+i d s)} b_{22} \\
& D_{4}=2 c \frac{|s| s[-s(d-b)-i(a+c)|s|]}{\left(c^{2}+d^{2}\right)(a|s|+i b s)} b_{22}
\end{aligned}
$$

Similarly, $C_{3}$ and $C_{4}$ can be expressed in terms of the auxiliary functions by using eqs. (22) and the results of eqs. (28) and (29).

Substituting expressions for the constants $C_{m}$ into the local stress equations (14) through (19) results in the formulation of a set of double integral equations with respect to the Fourier variables $s(-\infty<s<\infty)$ and $t(-a \leq t \leq a)$. Integrating with respect to $s$ will give a set of singular integral equations with respect to $t$, which are valid for any $j$ th crack within its own local coordinate system $\left(x_{j}, y_{j}\right)$ :

$$
\begin{aligned}
& \sigma_{x_{j} x_{j}}^{(j)}=\frac{1}{2 \pi} \int_{-a_{j}}^{a_{j}}\left[f_{j 1}\left(t_{j}\right) \frac{Q_{1}}{b_{11} Q_{0}}+f_{j 2}\left(t_{j}\right) \frac{Q_{2}}{b_{22} Q_{0}}\right] d t_{j} \\
& \sigma_{y_{j} y_{j}}^{(j)}=\frac{1}{2 \pi} \int_{-a_{j}}^{a_{j}}\left[f_{j 1}\left(t_{j}\right) \frac{Q_{3}}{b_{11} Q_{0}}+f_{j 2}\left(t_{j}\right) \frac{Q_{1}}{b_{22} Q_{0}}\right] d t_{j} \\
& \sigma_{x_{j} y_{j}}^{(j)}=\frac{1}{2 \pi} \int_{-a_{j}}^{a_{j}}\left[f_{j 1}\left(t_{j}\right) \frac{Q_{5}}{b_{11} Q_{0}}+f_{j 2}\left(t_{j}\right) \frac{Q_{6}}{b_{22} Q_{0}}\right] d t_{j}
\end{aligned}
$$

where

$$
\begin{gathered}
Q_{0}=a c\left[(a+c)^{2}+(b-d)^{2}\right]\left[\left(t_{j}-x_{j}\right)^{2}+2 b\left(t_{j}-x_{j}\right) y_{j}+\left(a^{2}+b^{2}\right) y_{j}^{2}\right] \\
\times\left[\left(t_{j}-x_{j}\right)^{2}+2 d\left(t_{j}-x_{j}\right) y_{j}+\left(c^{2}+d^{2}\right) y_{j}^{2}\right] \\
Q_{1}=R_{1}\left(t_{j}-x_{j}\right)^{3}+y_{j}\left[R_{2}\left(t_{j}-x_{j}\right)^{2}+y_{j} R_{3}\left(t_{j}-x_{j}\right)+y_{j}^{2} R_{4}\right]
\end{gathered}
$$

with 


$$
\begin{aligned}
R_{1}= & a^{2} b c+b^{3} c+2 a b c^{2}+2 a^{2} c d+a c^{2} d+a d^{3} \\
R_{2}= & a^{4} c+2 a^{2} b^{2} c+b^{4} c+2 a^{3} c^{2}+2 a b^{2} c^{2}+2 a^{2} c^{3}+a c^{4} \\
& +2 a^{2} b c d+2 b^{3} c d+2 a b c^{2} d+2 a^{2} c d^{2}+2 a c^{2} d^{2}+2 a b d^{3}+a d^{4} \\
R_{3}= & a^{2} b c^{3}+b^{3} c^{3}+2 a b c^{4}+2 a^{4} c d+4 a^{2} b^{2} c d+2 b^{4} c d+a^{3} c^{2} d \\
& +a b^{2} c^{2} d+a^{2} b c d^{2}+b^{3} c d^{2}+4 a b c^{2} d^{2}+a^{3} d^{3}+a b^{2} d^{3}+2 a b d^{4} \\
R_{4}= & \left(a^{2}+b^{2}\right)\left(c^{2}+d^{2}\right)\left(a^{2} c+b^{2} c+a c^{2}+a d^{2}\right) \\
Q_{2}= & R_{5}\left\{\left(t_{j}-x_{j}\right)^{3} R_{6}+y_{j}\left[R_{7}\left(t_{j}-x_{j}\right)^{2}-y_{j} R_{8}\left(t_{j}-x_{j}\right)+y_{j}^{2} R_{9}\right]\right\}
\end{aligned}
$$

with

$$
\begin{aligned}
R_{5}= & \left(a^{2}+b^{2}\right)\left(c^{2}+d^{2}\right) \\
R_{6}= & \left(a^{2} c+b^{2} c+a c^{2}+a d^{2}\right) \\
R_{7}= & a^{2} b c+b^{3} c+2 b^{2} c d+a c^{2} d+2 a b d^{2}+a d^{3} \\
R_{B}= & a^{3} c^{2}+a b^{2} c^{2}+a^{2} c^{3}-b^{2} c^{3}-2 a^{2} b c d-2 b^{3} c d \\
& -2 a b c^{2} d-a^{3} d^{2}-a b^{2} d^{2}+a^{2} c d^{2}-b^{2} c d^{2}-2 a b d^{3} \\
R_{9}= & \left(a^{2}+b^{2}\right)\left(c^{2}+d^{2}\right)(b c+a d) \\
Q_{3}= & R_{10}\left(t_{j}-x_{j}\right)^{3}+y_{j}\left[-\left(t_{j}-x_{j}\right)^{2} R_{11}+y_{j}\left(t_{j}-x_{j}\right) R_{12}+y_{j}^{2} R_{13}\right]
\end{aligned}
$$

with

$$
\begin{aligned}
R_{10} & =b c+a d \\
R_{11} & =\left(a^{2}-b^{2}\right) c-2 b d(a+c)+a\left(c^{2}-d^{2}\right) \\
R_{12} & =b c^{3}+a^{3} d+a b^{2} d+b c d^{2}+2 b d(b c+a d) \\
R_{13} & =\left(a^{2}+b^{2}\right)\left(c^{2}+d^{2}\right)(a+c)
\end{aligned}
$$

with

$$
\begin{aligned}
& R_{14}=a+c \\
R_{15} & =2 a b+b c+a d+2 c d \\
& R_{16}=a^{3}+a b^{2}+2 a^{2} c+2 a c^{2}+c^{3}+2 a b d+2 b c d+c d^{2} \\
& R_{17}=2 a b c^{2}+b c^{3}+a^{3} d+a b^{2} d+2 a^{2} c d+b c d^{2} \\
Q_{5}= & R_{18}\left(t_{j}-x_{j}\right)^{3}+y_{j}\left[R_{19}\left(t_{j}-x_{j}\right)^{2}-y_{j}\left(t_{j}-x_{j}\right) R_{20}+y_{j}^{2} R_{9}\right]
\end{aligned}
$$


with

$$
\begin{aligned}
R_{18}= & c\left(a^{2}+b^{2}\right)+a\left(c^{2}+d^{2}\right) \\
R_{19}= & a^{2} b c+b^{3} c+2 b^{2} c d+a c^{2} d+2 a b d^{2}+a d \\
R_{20}= & a^{3} c^{2}+a b^{2} c^{2}+a^{2} c^{3}-b^{2} c^{3}-2 a^{2} b c d \\
& -2 b^{3} c d-2 a b c^{2} d-a^{3} d^{2}-a b^{2} d^{2}+a^{2} c d^{2}-b^{2} c d^{2}-2 a b d^{3}
\end{aligned}
$$

and

$$
Q_{6}=R_{5}\left\{\left(t_{j}-x_{j}\right)^{3} R_{21}+y_{j}\left[R_{22}\left(t_{j}-x_{j}\right)^{2}+y_{j}\left(t_{j}-x_{j}\right) R_{23}+y_{j}^{2} R_{13}\right]\right\}
$$

with

$$
\begin{aligned}
& R_{21}=a d+b c \\
& R_{22}=-a^{2} c+b^{2} c-a c^{2}+a d^{2}+2 a b d+2 b c d \\
& R_{23}=b c^{3}+a^{3} d+a b^{2} d+2 b^{2} c d+2 a b d^{2}+b c d^{2}
\end{aligned}
$$

Note, that the special case of an isotropic material can be recovered by the following substitutions: $b=d=0$, and $a=c=1$, thus giving $R_{1}=R_{3}=R_{7}=R_{9}=R_{10}=R_{12}=$ $R_{15}=R_{17}=R_{19}=R_{21}=R_{23}=0 ; R_{5}=1 ; R_{4}=R_{6}=R_{8}=R_{11}=R_{13}=R_{14}=R_{18}=$ $R_{20}=-R_{22}=2 ;$ and $R_{2}=R_{16}=6$. The parameters $Q_{i}$ then become

$$
\begin{gathered}
Q_{0}^{(i s o)}=4\left[\left(t_{j}-x_{j}\right)^{2}+y_{j}^{2}\right]^{2} \\
Q_{1}^{(i s o)}=2 y_{j}\left[3\left(t_{j}-x_{j}\right)^{2}+y_{j}^{2}\right] \\
Q_{2}^{(i s o)}=2\left(t_{j}-x_{j}\right)\left[\left(t_{j}-x_{j}\right)^{2}-y_{j}^{2}\right] \\
Q_{3}^{(i s o)}=-2 y_{j}\left[\left(t_{j}-x_{j}\right)^{2}-y_{j}^{2}\right] \\
Q_{4}^{(i s o)}=2\left(t_{j}-x_{j}\right)\left[\left(t_{j}-x_{j}\right)^{2}+3 y_{j}^{2}\right] \\
Q_{5}^{(i s o)}=2\left(t_{j}-x_{j}\right)\left[\left(t_{j}-x_{j}\right)^{2}-y_{j}^{2}\right]=Q_{2}^{(i s o)} \\
Q_{6}^{(i s o)}=-2 y_{j}\left[\left(t_{j}-x_{j}\right)^{2}-y_{j}^{2}\right]=Q_{3}^{(i s o)}
\end{gathered}
$$

and the stresses reduce to the isotropic stress formulas derived previously in reference [2].

This completes the formulation of the fundamental problem (or local stress state) for the $j$ th crack. Henceforth, the formulation of the multiple crack problem will be addressed. 


\section{TOTAL STRESS FORMULATION}

The total stress state ${ }_{p}\left(\sigma_{r z}^{T}\right)$ for the pth crack is defined as the local stress state of the $p$ th crack $\left(\sigma_{F z}^{p}\right)$ plus the contribution to that stress state of all remaining cracks. This may be represented mathematically as

$$
{ }_{p} \sigma_{r z}^{T}\left(x_{p}, y_{p}\right)=\sigma_{r z}^{p}\left(x_{p}, y_{p}\right)+\sum_{j=1}^{n-1} \hat{\sigma}_{r z}^{j}\left[x_{j}\left(x_{p}, y_{p}\right), y_{j}\left(x_{p}, y_{p}\right)\right]
$$

for $p=1, \ldots, n$,where $\hat{\sigma}_{r z}^{j}$ is defined through standard tensor transformation of the stresses, i.e.,

$$
\hat{\sigma}_{r z}=\beta_{l r} \beta_{m z} \sigma_{l m}
$$

and $\beta_{l r}, \beta_{m z}$ are the direction cosines between the $\left(x_{j}, y_{j}\right)$ and $\left(x_{p}, y_{p}\right)$ coordinates with $j$ identifying the remaining cracks. Note, this statement does not imply that the concept of superposition has been invoked, since the stress perturbation boundary conditions (see eqs. (5)) have not yet been utilized to determine the unknown auxiliary functions.

For functional compatibility within eq. (50), coordinate transformations must be simultaneously applied to all remaining $j$ th crack coordinate variables. As a result, the dominant part (i.e., the first term of eq. (50)) possesses a singularity whereas the regular terms within the summation lose their original singularities and yet still contribute to the total stress state, as one might expect.

By replacing subscript $j$ by $p$ in eqs. (33 and 32), respectively, and evaluating them at $y_{p}=0$, the singular terms of the singular integral equations are obtained from the first term of eq. (50) applied for shear $\left(\sigma_{x y}\right)$ and for normal stress $\left(\sigma_{y y}\right)$. Finally, the variables $x$ and $t$ are normalized by using $x_{p}=a_{p} \xi$ and $t_{p}=a_{p} \tau$, where $\xi$ and $\tau$ are defined between -1 and 1. Therefore,

$$
\begin{aligned}
& \sigma_{x_{p} y_{p}}^{(p)}=\frac{1}{\pi} \int_{-1}^{1}\left[E_{x y}^{p(1)} \frac{f_{p 1}\left(\tau_{p}\right)}{\tau_{p}-\xi_{p}}+E_{x y}^{p(2)} \frac{f_{p 2}\left(\tau_{p}\right)}{\tau_{p}-\xi_{p}}\right] d \tau_{p} \\
& \sigma_{y y_{p}}^{(p)}=\frac{1}{\pi} \int_{-1}^{1}\left[E_{v y}^{p(1)} \frac{f_{p 1}\left(\tau_{p}\right)}{\tau_{p}-\xi_{p}}+E_{y y}^{p(2)} \frac{f_{p 2}\left(\tau_{p}\right)}{\tau_{p}-\xi_{p}}\right] d \tau_{p}
\end{aligned}
$$

where $E_{x y}$ and $E_{y y}$ are material-related coefficients proportional to local $x$-direction stiffness (denoted by superscript 1) or local $y$-direction stiffness (denoted by superscript 2) with respect to the local crack coordinate system. Consequently, we will call them modified stiffness parameters (MSP's). The MSP's are

$$
\begin{aligned}
& E_{x y}^{p(1)}=\frac{\left[c\left(a^{2}+b^{2}\right)+a\left(c^{2}+d^{2}\right)\right]}{2 a c\left[(a+c)^{2}+(b-d)^{2}\right] b_{11}} \\
& E_{x y}^{p(2)}=\frac{\left(a^{2}+b^{2}\right)\left(c^{2}+d^{2}\right)(a d+b c)}{2 a c\left[(a+c)^{2}+(b-d)^{2}\right] b_{22}} \\
& E_{y y}^{p(1)}=\frac{(b c+a d)}{2 a c\left[(a+c)^{2}+(b-d)^{2}\right] b_{11}} \\
& E_{y y}^{p(2)}=\frac{\left(a^{2}+b^{2}\right)\left(c^{2}+d^{2}\right)(a+c)}{2 a c\left[(a+c)^{2}+(b-d)^{2}\right] b_{22}}
\end{aligned}
$$




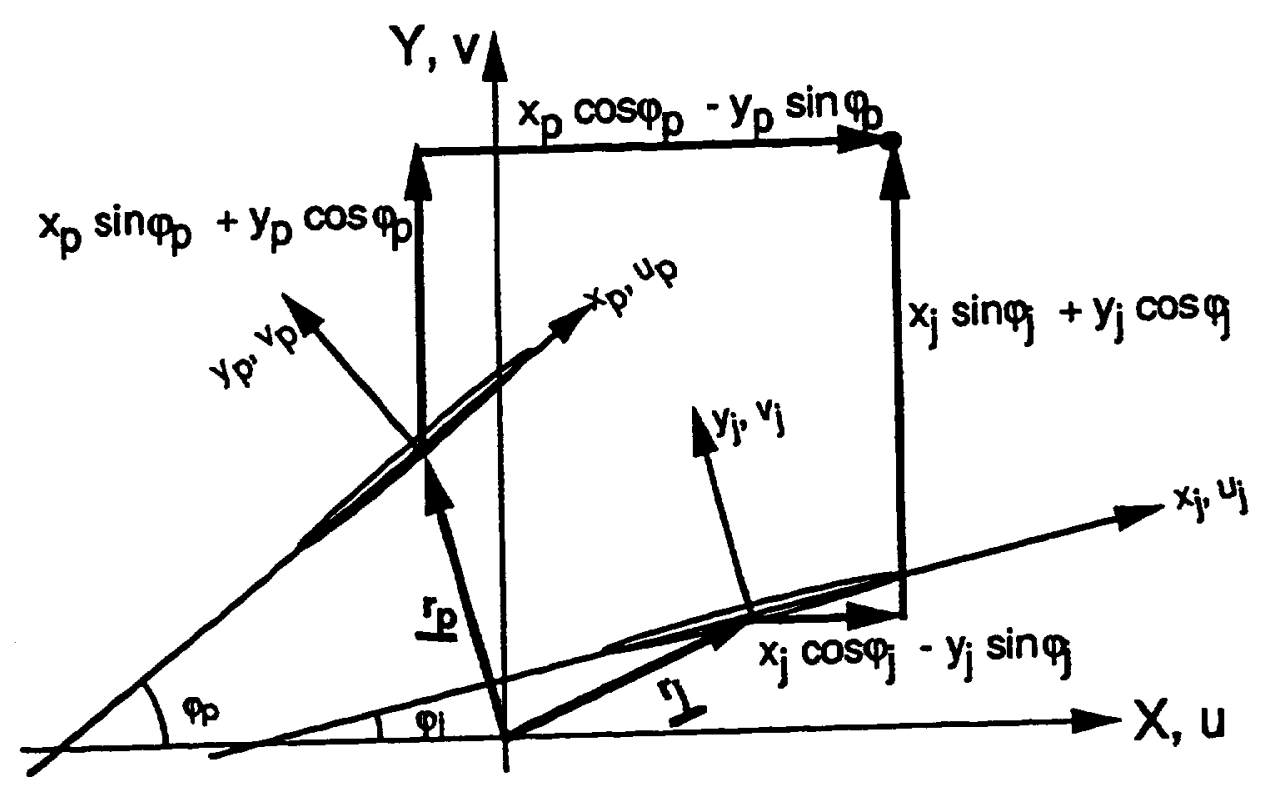

Figure 2: Geometric relationships between a pair of cracks and their local variables.

For the isotropic case

$$
E_{x y}^{p(2)}=E_{y y}^{p(1)}=0
$$

and

$$
E_{x y}^{p(1)}=E_{v y}^{p(2)}=\frac{E}{4}
$$

whereas in the orthotropic case, only

$$
E_{x y}^{p(2)}=E_{y y}^{p(1)}=0
$$

The regular terms of the singular integral equations are obtained by transforming the remaining stresses into the local pth crack coordinate system simultaneously with coordinate transformation. The coordinate transformation between the $x_{j}, y_{j}$ and $x_{p}, y_{p}$ systems is determined from the following geometric relationship (see Fig. 2):

$$
\begin{aligned}
& r_{j X}+x_{j} \cos \varphi_{j}-y_{j} \sin \varphi_{j}=r_{p X}+x_{p} \cos \varphi_{p}-y_{p} \sin \varphi_{p} \\
& r_{j Y}+x_{j} \sin \varphi_{j}+y_{j} \cos \varphi_{j}=r_{p Y}+x_{p} \sin \varphi_{p}+y_{p} \cos \varphi_{p}
\end{aligned}
$$

where $r_{j X}, r_{j Y}$ are the rectangular components of the $j$ th crack position vector referred to the global coordinate system $X-Y$, and $\varphi_{j}$ is the angle of rotation between the global and local systems.

One component of the regular part of the total stress is obtained by transformation of the stresses from $j$ th crack local coordinate system into the pth crack local coordinate system simultaneously with the coordinate transformation and substituting $y_{p}=0$ :

$$
\begin{gathered}
\hat{\sigma}_{x_{p} y_{p}}^{j}=-\left(\sigma_{x_{j} x_{j}}^{(j)}-\sigma_{y_{j} y_{j}}^{(j)}\right) \sin \theta \cos \theta+\sigma_{x_{j} y_{j}}^{(j)}\left(\cos ^{2} \theta-\sin ^{2} \theta\right) \\
\hat{\sigma}_{y_{p} y_{p}}^{j}=\sigma_{x_{j} x_{j}}^{(j)} \sin ^{2} \theta+\sigma_{y_{j} y_{j}}^{(j)} \cos ^{2} \theta-2 \sigma_{x_{j} y_{j}}^{(j)} \sin \theta \cos \theta
\end{gathered}
$$


where $\theta=\varphi_{p}-\varphi_{j}$. Therefore, we can obtain from eqs. (58) and (59)

$$
\begin{aligned}
& x_{j}=p_{1}+x_{p} \cos \theta \\
& y_{j}=p_{2}+x_{p} \sin \theta
\end{aligned}
$$

where $(p 1, p 2)$ is the vector connecting the centers from $j$ th to $p$ th cracks expressed in the $j$ th coordinate system:

$$
\begin{aligned}
& p_{1}=\left(r_{p Y}-r_{j Y}\right) \sin \varphi_{j}+\left(r_{p X}-r_{j X}\right) \cos \varphi_{j} \\
& p_{2}=\left(r_{p Y}-r_{j Y}\right) \cos \varphi_{j}-\left(r_{p X}-r_{j X}\right) \sin \varphi_{j}
\end{aligned}
$$

The regular, normalized form of the parameters $Q_{i}$ for $i=0,1, \ldots, 6$ is obtained by using the coordinate normalization $x_{p}=a_{p} \xi$ and $t_{j}=a_{j} \tau$, in addition to the coordinate transformation, to produce the parameters $Q_{i}^{(\text {reg) }}$ :

$$
\begin{aligned}
& Q_{0}^{(r e g)}=a c\left[(a+c)^{2}+(b-d)^{2}\right]\left[\left(a_{j} \tau-p_{1}-a_{p} \xi \cos \theta\right)^{2}+\right. \\
& \left.2 b\left(a_{j} \tau-p_{1}-a_{p} \xi \cos \theta\right)\left(p_{2}+a_{p} \xi \sin \theta\right)+\left(a^{2}+b^{2}\right)\left(p_{2}+a_{p} \xi \sin \theta\right)^{2}\right] \\
& {\left[\left(a_{j} \tau-p_{1}-a_{p} \xi \cos \theta\right)^{2}+2 d\left(a_{j} \tau-p_{1}-a_{p} \xi \cos \theta\right)\left(p_{2}+a_{p} \xi \sin \theta\right)+\right.} \\
& \left.\left(c^{2}+d^{2}\right)\left(p_{2}+a_{p} \xi \sin \theta\right)^{2}\right] \\
& \begin{aligned}
Q_{1}^{(\text {reg })}= & R_{1}\left(a_{j} \tau-p_{1}-a_{p} \xi \cos \theta\right)^{3}+\left(p_{2}+a_{p} \xi \sin \theta\right)\left[R_{2}\left(a_{j} \tau-p_{1}-a_{p} \xi \cos \theta\right)^{2}\right. \\
& \left.+\left(p_{2}+a_{p} \xi \sin \theta\right) R_{3}\left(a_{j} \tau-p_{1}-a_{p} \xi \cos \theta\right)+\left(p_{2}+a_{p} \xi \sin \theta\right)^{2} R_{4}\right]
\end{aligned} \\
& \begin{aligned}
Q_{2}^{(\text {reg })}= & \left\{\left(a_{j} \tau-p_{1}-a_{p} \xi \cos \theta\right)^{3} R_{6}+\left(p_{2}+a_{p} \xi \sin \theta\right)\left[R_{7}\left(a_{j} \tau-p_{1}-a_{p} \xi \cos \theta\right)^{2}\right.\right. \\
& \left.\left.-\left(p_{2}+a_{p} \xi \sin \theta\right) R_{8}\left(a_{j} \tau-p_{1}-a_{p} \xi \cos \theta\right)+\left(p_{2}+a_{p} \xi \sin \theta\right)^{2} R_{0}\right]\right\} R_{5}
\end{aligned} \\
& \begin{aligned}
Q_{3}^{(r e g)}= & R_{10}\left(a_{j} \tau-p_{1}-a_{p} \xi \cos \theta\right)^{3}+\left(p_{2}+a_{p} \xi \sin \theta\right)\left[-\left(a_{j} \tau-p_{1}-a_{p} \xi \cos \theta\right)^{2} R_{11}\right. \\
& \left.+\left(p_{2}+a_{p} \xi \sin \theta\right)\left(a_{j} \tau-p_{1}-a_{p} \xi \cos \theta\right) R_{12}+\left(p_{2}+a_{p} \xi \sin \theta\right)^{2} R_{9}\right]
\end{aligned} \\
& \begin{aligned}
Q_{4}^{(r e g)}= & \left\{\left(a_{j} \tau-p_{1}-a_{p} \xi \cos \theta\right)^{3} R_{14}+\left(p_{2}+a_{p} \xi \sin \theta\right)\left[R_{15}\left(a_{j} \tau-p_{1}-a_{p} \xi \cos \theta\right)^{2}\right.\right. \\
& \left.\left.+\left(p_{2}+a_{p} \xi \sin \theta\right)\left(a_{j} \tau-p_{1}-a_{p} \xi \cos \theta\right) R_{16}+\left(p_{2}+a_{p} \xi \sin \theta\right)^{2} R_{17}\right]\right\} R_{5}
\end{aligned} \\
& Q_{5}^{(\text {reg })}=R_{18}\left(a_{j} \tau-p_{1}-a_{p} \xi \cos \theta\right)^{3}+\left(p_{2}+a_{p} \xi \sin \theta\right)\left[R_{19}\left(a_{j} \tau-p_{1}-a_{p} \xi \cos \theta\right)^{2}\right. \\
& \left.-\left(p_{2}+a_{p} \xi \sin \theta\right)\left(a_{j} \tau-p_{1}-a_{p} \xi \cos \theta\right) R_{20}+\left(p_{2}+a_{p} \xi \sin \theta\right)^{2} R_{0}\right] \\
& \begin{aligned}
Q_{6}^{(r e g)}= & \left\{\left(a_{j} \tau-p_{1}-a_{p} \xi \cos \theta\right)^{3} R_{21}+\left(p_{2}+a_{p} \xi \sin \theta\right)\left[R_{22}\left(a_{j} \tau-p_{1}-a_{p} \xi \cos \theta\right)^{2}\right.\right. \\
& \left.\left.+\left(p_{2}+a_{p} \xi \sin \theta\right)\left(a_{j} \tau-p_{1}-a_{p} \xi \cos \theta\right) R_{23}+\left(p_{2}+a_{p} \xi \sin \theta\right)^{2} R_{13}\right]\right\} R_{5}
\end{aligned}
\end{aligned}
$$


So the regular normalized component of the shear stress becomes

$$
\hat{\sigma}_{x_{p} y_{p}}^{j}=\int_{-1}^{1} \operatorname{ker}_{1} f_{j 1}\left(t_{j}\right) d \tau_{j}+\int_{-1}^{1} \operatorname{ker}_{2} f_{j 2}\left(t_{j}\right) d \tau_{j}
$$

where

$$
\begin{aligned}
& \operatorname{ker}_{1}=\frac{a_{j}}{2 \pi} \frac{1}{b_{11} Q_{0}^{(r e g)}}\left[-\left(Q_{1}^{(\text {reg })}-Q_{3}^{(\text {reg })}\right) \sin \theta \cos \theta+Q_{5}^{(r e g)}\left(\cos ^{2} \theta-\sin ^{2} \theta\right)\right] \\
& \operatorname{ker}_{2}=\frac{a_{j}}{2 \pi} \frac{1}{b_{22} Q_{0}^{(\text {reg })}}\left[-\left(Q_{2}^{(\text {reg) }}-Q_{4}^{(\text {reg })}\right) \sin \theta \cos \theta+Q_{6}^{(\text {reg })}\left(\cos ^{2} \theta-\sin ^{2} \theta\right)\right]
\end{aligned}
$$

The regular normalized component of normal stress is

$$
\hat{\sigma}_{y_{p y p}}^{j}=\int_{-1}^{1} \operatorname{ker}_{3} f_{j 1}\left(t_{j}\right) d \tau_{j}+\int_{-1}^{1} \operatorname{ker}_{4} f_{j 2}\left(t_{j}\right) d \tau_{j}
$$

where

$$
\begin{aligned}
& \operatorname{ker}_{3}=\frac{a_{j}}{2 \pi} \frac{1}{b_{11} Q_{0}^{(r e g)}}\left[Q_{1}^{(r e g)} \sin ^{2} \theta+Q_{3}^{(r e g)} \cos ^{2} \theta-2 Q_{5}^{(r e g)} \sin \theta \cos \theta\right] \\
& \operatorname{ker}_{4}=\frac{a_{j}}{2 \pi} \frac{1}{b_{22} Q_{0}^{(r e g)}}\left[Q_{2}^{(r e g)} \sin ^{2} \theta+Q_{4}^{(r e g)} \cos ^{2} \theta-2 Q_{6}^{(r e g)} \sin \theta \cos \theta\right]
\end{aligned}
$$

Thus, the total stresses $\left(\sigma_{y y}^{T}\right.$ and $\sigma_{x y}^{T}$ ) for $n$ cracks can be written in the following form:

$$
\begin{aligned}
{ }_{n} \sigma_{x y}^{T} & =\left\{\int_{-1}^{1} \operatorname{ker}_{1} f_{11} d \tau+\int_{-1}^{1} \operatorname{ker}_{2} f_{12} d \tau+\ldots+\int_{-1}^{1} \operatorname{ker}_{1} f_{(n-1) 1} d \tau\right. \\
& \left.+\int_{-1}^{1} \operatorname{ker}_{2} f_{(n-1) 2} d \tau+\frac{E_{s x}^{n(1)}}{\pi} \int_{-1}^{1} \frac{f_{n 1}}{\tau-\xi} d \tau+\frac{E_{\pi x}^{n(2)}}{\pi} \int_{-1}^{1} \frac{f_{n 2}}{\tau-\xi} d \tau\right\} \\
{ }_{n} \sigma_{y y}^{T} & =\left\{\int_{-1}^{1} \operatorname{ker}_{3} f_{11} d \tau+\int_{-1}^{1} \operatorname{ker}_{4} f_{12} d \tau+\ldots+\int_{-1}^{1} \operatorname{ker}_{3} f_{(n-1) 1} d \tau\right. \\
& \left.+\int_{-1}^{1} \operatorname{ker}_{4} f_{(n-1) 2} d \tau+\frac{E_{n(1)}^{n(1)}}{\pi} \int_{-1}^{1} \frac{f_{n 1}}{\tau-\xi} d \tau+\frac{E_{n x}^{n(2)}}{\pi} \int_{-1}^{1} \frac{f_{n 2}}{\tau-\xi} d \tau\right\}
\end{aligned}
$$

The formulation of this system of singular integral equations is complete once the single-value conditions for the auxiliary functions $f_{j \eta}$ are chosen. In the case of straight cracks, this single-value condition [3] is

$$
\int_{-1}^{1} f_{j \eta}(\tau) d \tau=0
$$

where $j$ stands for the $j$ th crack, and $\eta$ takes on the value of 1 or 2 .

\section{SOLUTION FOR THE STRESS INTENSITY FAC- TORS}

The integral equations obtained are of the Cauchy type; thus for sharp cracks, the stresses and strains will have a square-root singularity, and the classic definition of a SIF may be used (see refs. [4], [5], [6] and [7]). Therefore, the mode I and II SIF's for the $j$ th crack are 


$$
\begin{aligned}
k_{1}^{j}(1) & =\lim _{\xi \rightarrow 1}[2(\xi-1)]^{\frac{1}{2}}\left\{j \sigma_{y y}^{T}(\xi, 0)\right\} \\
k_{2}^{j}(1) & =\lim _{\xi \rightarrow 1}[2(\xi-1)]^{\frac{1}{2}}\left\{j \sigma_{x y}^{T}(\xi, 0)\right\} \\
k_{1}^{j}(-1) & =\lim _{\xi \rightarrow-1}[-2(1+\xi)]^{\frac{1}{2}}\left\{j \sigma_{y y}^{T}(\xi, 0)\right\} \\
k_{2}^{j}(-1) & =\lim _{\xi \rightarrow-1}[-2(1+\xi)]^{\frac{1}{2}}\left\{j \sigma_{x y}^{T}(\xi, 0)\right\}
\end{aligned}
$$

where the normal and shear stresses, eqs. (76) and (77), are used. Note that this definition of a SIF was originally developed for an isotropic material wherein the modeI normal stress is related only to the normal crack-opening displacement $\Delta v$ and the mode-II shear stress is related only to the shear displacement $\Delta u$. The same definition can also be applied to anisotropic materials by assuming that modes I and II are based on normal and shear stresses only; however, as will be shown in egs. (86) to (89), these stresses are driven by a mixed mode displacement $(\Delta v$ and $\Delta u)$ field.

It is well known [3] that the auxiliary functions $(f)$ can be expressed as a product of the unknown bounded functions $(H)$ and the known singular weight functions $(w)$ :

$$
f(\tau)=H(\tau) w(\tau)
$$

The singular weight function $w$ for a sharp crack is

$$
w(\tau)=\left(\tau^{2}-1\right)^{-\frac{1}{2}}
$$

Erdogan [3] found, for example, that in the case of a Cauchy-type singular integral equation (eqs. (76) and (77)), the dominant part can be expressed in terms of the function $H$ evaluated at the tips of the $j$ th crack:

$$
\frac{1}{\pi} \int_{-1}^{1} \frac{f_{\eta j} d \tau}{\tau-\xi}=H_{\eta j}(-1) \frac{e^{i \pi / 2}}{\sqrt{2}}(\tau+1)^{-\frac{1}{2}}-H_{\eta j}(1) \frac{1}{\sqrt{2}}(\tau-1)^{-\frac{1}{2}}+O(\tau)
$$

where $\eta$ is 1 or 2 , and $O(\tau)$ is the higher order term, which in subsequent calculations is neglected. Equations (85) can be substituted for the dominant part (last two terms in eqs. (76) and (77)) of the normal and shear components of the total stresses in eqs. (79) to (82). This substitution and subsequent evaluation of the limits at the crack tips results in the redefining of the SIF's, normalized with respect to $\sqrt{a_{1}}$ and $\sigma_{j p}^{\circ}$, and expressed in terms of the functions $H_{\eta j}$ :

$$
\begin{gathered}
k_{1}^{j}(1)=\left[E_{y y}^{j(1)} H_{1 j}(1)+E_{y y}^{j(2)} H_{2 j}(1)\right] \sqrt{a_{j} / a_{1}} \\
k_{2}^{j(1)}=\left[E_{x y}^{j(1)} H_{1 j}(1)+E_{x y}^{j(2)} H_{2 j}(1)\right] \sqrt{a_{j} / a_{1}} \\
k_{1}^{j}(-1)=-\left[E_{y y}^{j(1)} H_{1 j}(-1)+E_{y y}^{j(2)} H_{2 j}(-1)\right] \sqrt{a_{j} / a_{1}} \\
k_{2}^{j}(-1)=-\left[E_{x y}^{j(1)} H_{1 j}(-1)+E_{x y}^{j(2)} H_{2 j}(-1)\right] \sqrt{a_{j} / a_{1}}
\end{gathered}
$$

The Lobatto-Chebyshev collocation integration technique was employed, because it is known to provide excellent results in dealing with the preceding Cauchy-type singular 
integral equations. The unknown functions $H_{\eta j}$ are determined at a discrete set of points $\tau_{1}, \tau_{2}, \ldots, \tau_{m}$ called abscissas. In this way, each integral equation is reduced to a set of algebraic equations with unknowns $H_{\eta j}\left(\tau_{1}\right), H_{\eta j}\left(\tau_{2}\right), \ldots, H_{\eta j}\left(\tau_{m}\right)$, which are the discrete values of the functions $H_{\eta j}$; hence its name, discrete auxiliary function. Note that $H_{1 j}$ and $H_{2 j}$ are proportional, respectively, to the difference in shear and normal displacements at the crack tips:

$$
\begin{aligned}
& \Delta u \sim H_{1} \\
& \Delta v \sim H_{2}
\end{aligned}
$$

Consequently, $H_{\eta j}$ can be used as a measure of the crack-opening displacement at the crack tip.

Each of the singular integral equations subjected to the stress boundary conditions (eqs. 5) can be replaced by $m-1$ algebraic equations with $2 \mathrm{~nm}$ unknown parameters (see ref. [2]).In the Lobatto-Chebyshev method, the abscissas are calculated according to

$$
\tau_{r}=\cos \frac{(r-1) \pi}{m-1} \text { for } \quad r=1, \ldots, m
$$

with the corresponding weights given by

$$
w_{1}=w_{m}=\frac{\pi}{2(m-1)} \text { and } w_{r}=\frac{\pi}{m-1} \text { for } r=2,3, \ldots, m-1
$$

The collocation points are then found by using the formula

$$
\xi_{2}=\cos \frac{(2 z-1) \pi}{2 m-2} \text { for } z=1,2, \ldots, m-1
$$

In order to have the complete system of $2 \mathrm{~nm}$ algebraic equations, the single-value conditions (eqs.(78)) are also expressed by using the collocation technique:

$$
\sum_{r=1}^{m} H_{\eta j}\left(\tau_{r}\right) w_{r}=0
$$

Thus, the resulting system of algebraic equations can be written in the form

$$
[A]\{H\}=\{\mathcal{R}\}
$$

where [A] is a fully populated $2 n m \times 2 n m$ matrix of coefficients and $\{\mathcal{R}\}$ is the loading function vector.

The unknown parameter vector $\{H\}$ can be determined through inversion of the $[\mathrm{A}]$ matrix; thus,

$$
\{H\}=[A]^{-1}\{\mathcal{R}\}
$$

although only the appropriate values (i.e., $H_{\eta j}( \pm 1)$ ) are used to calculate the SIF's for the $j$ th crack (see eqs. (86) to (89)).

The general solution for any multicrack problem is now complete with the automatic generation of the associated FORTRAN code for the evaluation of eqs. (96). This FORTRAN program was utilized to obtained the following results, which are compared with published results obtained by other methods. 


\section{STRAIN ENERGY RELEASE RATE}

In fracture mechanics, perhaps the most important physical quantity is the strain energy release rate $\left(G_{T}\right)$, usually denoted by $G$. Cherepanov[8] discussed the generalized formula for $G_{T}$ given an anisotropic material.

$$
\begin{gathered}
G=\frac{1}{2} \int_{a}^{a+d a}\left\{\sigma_{v v}^{T}(x, 0)\left[v\left(x-d a, 0^{+}\right)-v\left(x-d a, 0^{-}\right)\right]+\right. \\
\left.\sigma_{x}^{T}(x, 0)\left[u\left(x-d a, 0^{+}\right)-u\left(x-d a, 0^{-}\right)\right]\right\} d x
\end{gathered}
$$

Using the roots of the characteristic equation in terms of the real components eq. (11), we can show that

$$
G_{T}=\frac{\pi}{2}\left[k_{1}^{2} \frac{(a+c)(a c-b d)+(b+d)(a d+b c)}{(a c-b d)^{2}+(a d+b c)^{2}} b_{22}+k_{2}^{2}(a+c) b_{11}\right]
$$

or utilizing eqs. (102) and (105), developed subsequently, we can obtain a simplified expression for $\mathrm{G}_{\mathrm{T}}$, that is

$$
G_{T}=\frac{\pi}{2} K_{e f f}^{2}(a+c) b_{11}
$$

where

$$
K_{e f f}=\sqrt{k_{1}^{2} \frac{E_{x y}^{(1)}}{E_{y y}^{(2)}}+k_{2}^{2}}
$$

and represents an effective SIF. Note the significant coupling between the normal and shear stresses and the displacement components in this effective SIF.

In the case of an isotropic material we may substitute $a=c=1, b=d=0$, $E_{x y}^{(1)}=E_{y y}^{(2)}$, and $b_{11}=b_{22}=\frac{1}{E}$ into eq. (98). Consequently, the well-known fracture mechanics relationship is recovered:

$$
G^{(i s 0)}=\frac{\pi}{E}\left\{\left[k_{1}^{(i s 0)}\right]^{2}+\left[k_{2}^{(i s o)}\right]^{2}\right\}
$$

\section{NUMERICAL APPLICATIONS}

The focus of the following parametric study will be limited to investigating the influence of the crack geometry configuration and strength of anisotropy on the resulting driving force. To accomplish this the four independent elastic constants were taken to be

$$
\begin{aligned}
& \frac{G_{L T}}{E_{T T}}=0.4 \\
& v_{L T}=0.25 \\
& \frac{E_{T T}}{E_{T T}}=1.0
\end{aligned}
$$

and the strength of anisotropy, $\frac{E_{x h}}{E_{T T}}$, is a specified constant greater than or equal to one. Note that $\mathrm{E}_{T T}$ is always chosen to be the weaker direction, i.e., to be less than or equal to $\mathrm{E}_{L L}$. Although, the influence of $G_{L T}$ and $v_{L T}$ on the driving force is important, this aspect of the parametric study will be reserved for future work. 


\subsection{Two Crack Interaction}

\subsubsection{Collinear Cracks}

In order to validate the results obtained with the automatically generated FORTRAN code, the well-known problem of two parallel interacting cracks is considered here. The plate, with two cracks of length $2 a$ and a preferred direction defined by $\Omega=22^{\circ}$, is subjected to a normal far-field stress state $\left(\sigma_{Y Y}^{\circ}\right)$ as shown in Fig. 3. Results are obtained

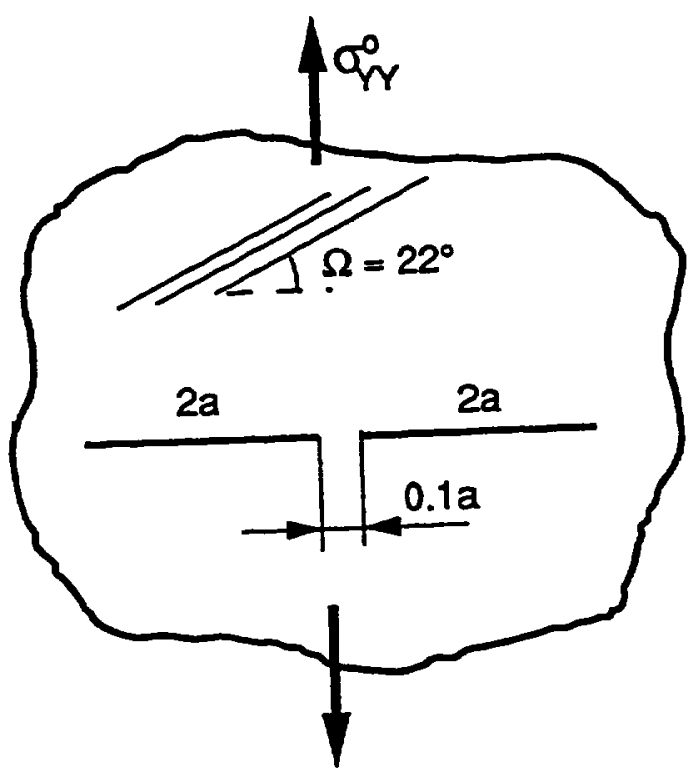

Figure 3: Geometry and loading condition of two collinear cracks problem.

over a wide range of strengths of anisotropy, as defined by the ratio $E_{L L} / E_{T T}$. As Fig. 4 shows, the SIF's do not depend on the strength of anisotropy, even though the discrete auxiliary functions do. The results indicate that at both the inner and outer crack tips mode-I SIF's are exactly the same as the isotropic SIF's from references [9] and [11], and mode-II SIF's are zero for this configuration.

Although, the mode-II SIF is zero over the entire range of strengths of anisotropy examined, the shear crack opening, as represented by $H_{1}$ (see eq. (90) is zero only for the special case of an isotropic material (i.e., $E_{L L} / E_{T T}=1$ ). Figure 4 clearly shows that even small amounts of anisotropy $\left(E_{L L} / E_{T T}>1\right)$ produce shear displacements at the crack tip and that this shear displacement increases significantly for $1<E_{L L} / E_{T T}<5$ and becomes constant for $E_{L L} / E_{T T}>15$. Consequently, two collinear cracks within a transversely isotropic material will always (provided $\Omega=22^{\circ}$ ) produce a mode-I crack tip local stress field with a mixed-mode local displacement field even when the strength of anisotropy is small. This fact can be understood, even for the case of a single crack[10], by examining eqs. (86) to (89), where it is apparent that in an anisotropic material the normal and shear stresses are coupled with both normal and shear displacement components, respectively. 


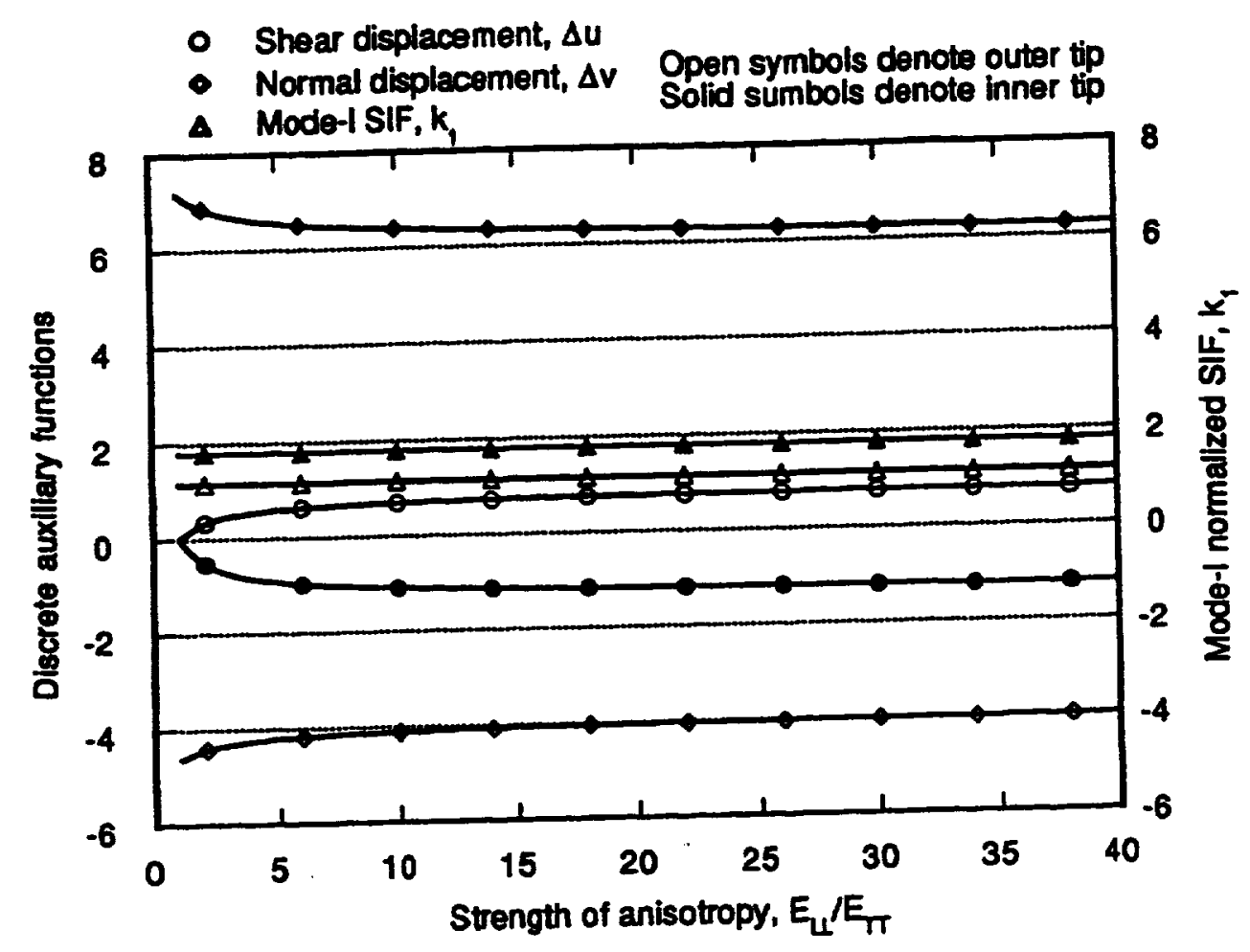

Figure 4: Discrete auxiliary functions and normalized SIF's versus strength of anisotropy $E_{L L} / E_{T T}$ for two equal collinear cracks in a composite plate reinforced at $\Omega=22^{\circ}$ and subjected to far-field normal stress, see Fig. 3.

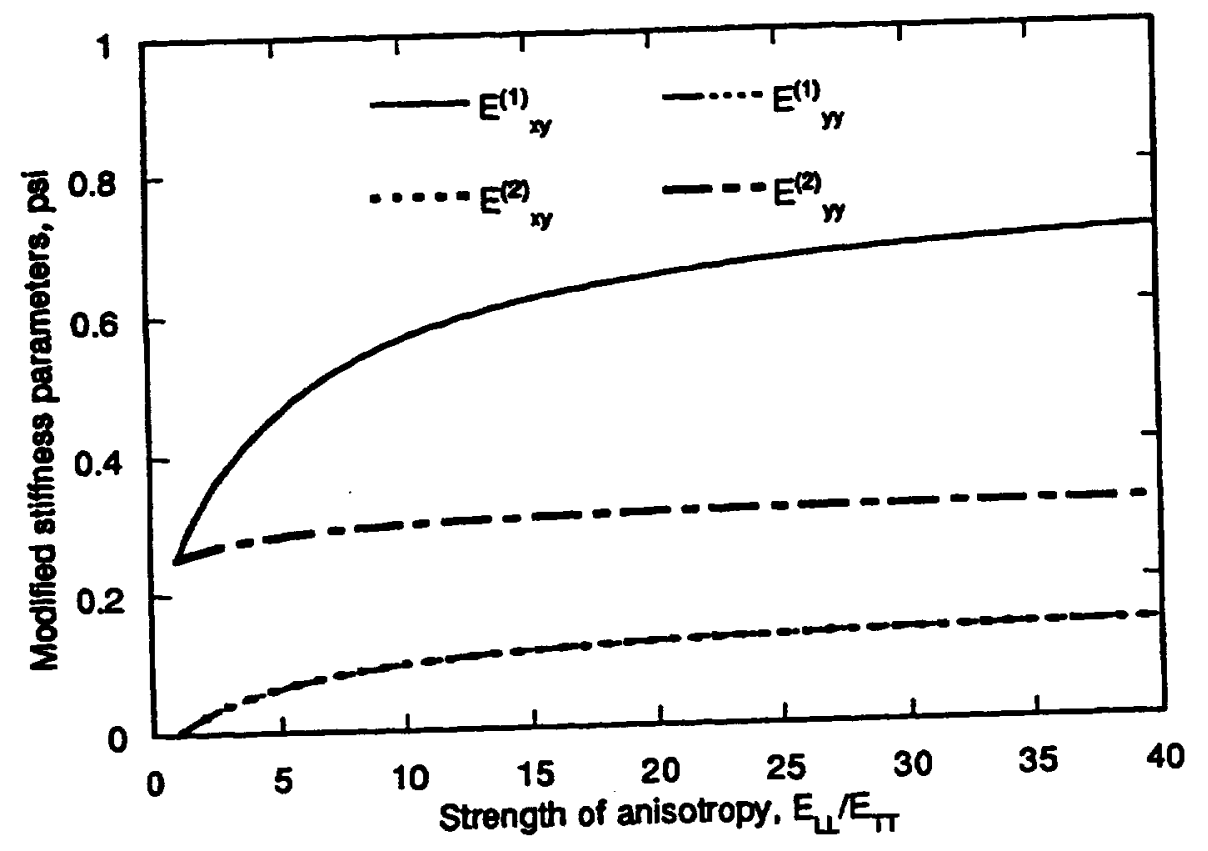

Figure 5: Modified stiffness parameters versus strength of anisotropy $E_{L L} / E_{T T}$ for two equal collinear cracks in a composite plate reinforced at $\Omega=22^{\circ}$ and subjected to far-field normal stress, see Fig. 3. 


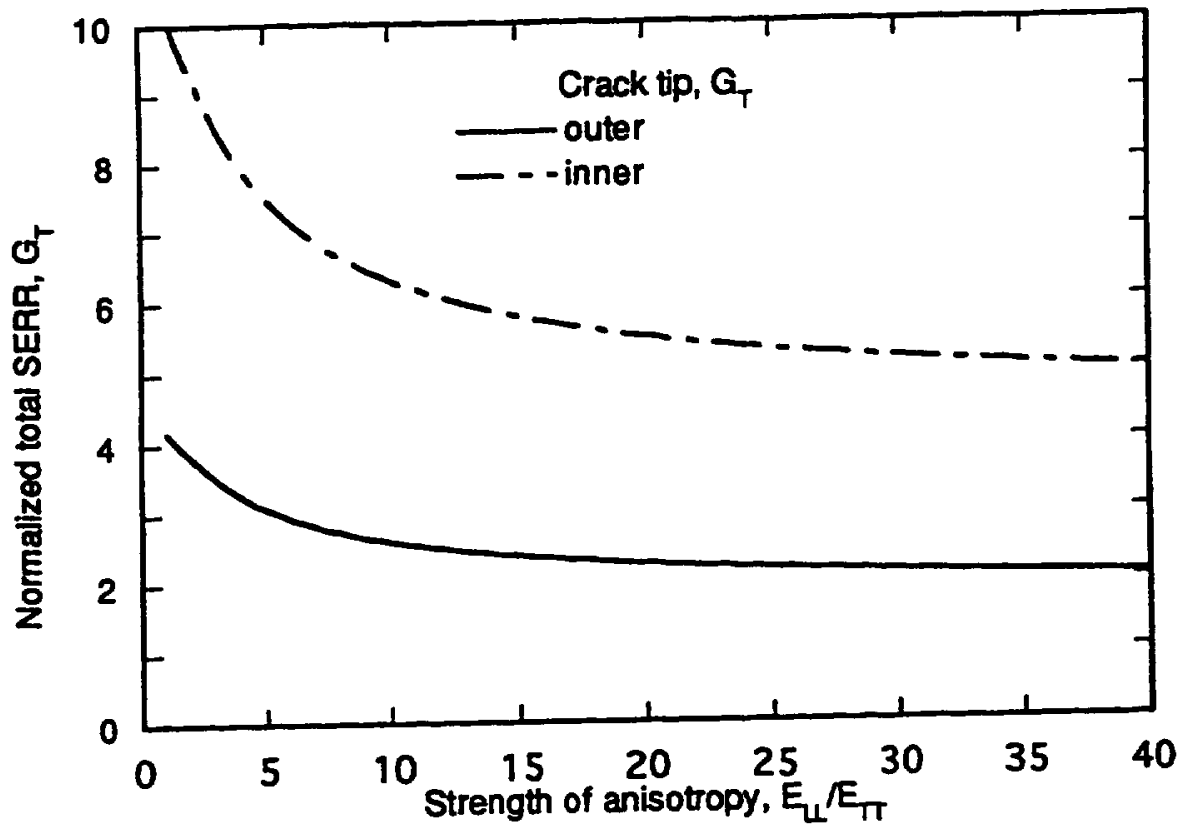

Figure 6: Normalized $\mathrm{G}_{T}$ versus strength of anisotropy $E_{L L} / E_{T T}$ for two equal collinear cracks in a composite plate (reinforced at $\Omega=22^{\circ}$; and subjected to far-field normal stress, see Fig. 3).

Figure 5 shows how the MSP's vary with respect to the strength of anisotropy $E_{L L} / E_{T T}$. Using the isotropic values (i.e., $E_{L L} / E_{T T}=1$ ) of the MSP's, we can obtain the results given in equation (57). Increasing the strength of anisotropy significantly increases $E_{x y}^{(1)}, E_{x y}^{(2)}$ and $E_{y y}^{(1)}$, while $E_{y y}^{(2)}$ remains nearly constant. Note that $E_{x y}^{(2)}=E_{y y}^{(1)}$ over the entire range of $E_{L L} / E_{T T}$. Revisiting eq. (87) and Figs 4 and 5, explains why the mode II SIF is zero. It stems from the fact that although both normal and shear displacements are induced, $E_{x y}^{(2)}$ is so much less than $E_{x y}^{(1)}$ that the influences of the normal and shear displacements are counteracted.

As discussed previously, the total $\mathrm{G}_{T}$ represents an important measure of the driving force for crack propagation in fracture mechanics. The $G_{T}$ 's at the inner and outer crack tips for the two collinear cracks are shown in Fig. 6. Clearly, the maximum $\mathrm{G}_{T}$ 's (for both the inner and outer crack tips) occur in the isotropic case. In the anisotropic case when $E_{L L} / E_{T T}>15$ the $G_{T}$ 's rapidly reduce to nearly $50 \%$ of the isotropic values. Although both the $G_{T}$ (Fig. 6) and the SIF's (Fig. 4) indicate that the inner crack tip will propagate, only the $G_{T}$ 's unambiguously indicate that as the strength of anisotropy increases, the crack-driving force is reduced. Thus, we can conclude that (1) the isotropic case gives rise to the greatest driving force and (2) that the SIF (unlike the $G_{T}$ ) is unable to detect the decrease in the crack-driving force as a function of strength of anisotropy.

Now let us examine the influence of changing the preferred direction angle $\Omega$ on the same collinear crack configuration with a strength of anisotropy ratio $E_{L L} / E_{T T}=40$. Results shown in Fig. 7 indicate that the mode-I SIF's are the same as those previously obtained, even though the discrete auxiliary functions show that the normal and shear crack-opening displacements vary significantly with preferred direction. Note that the 


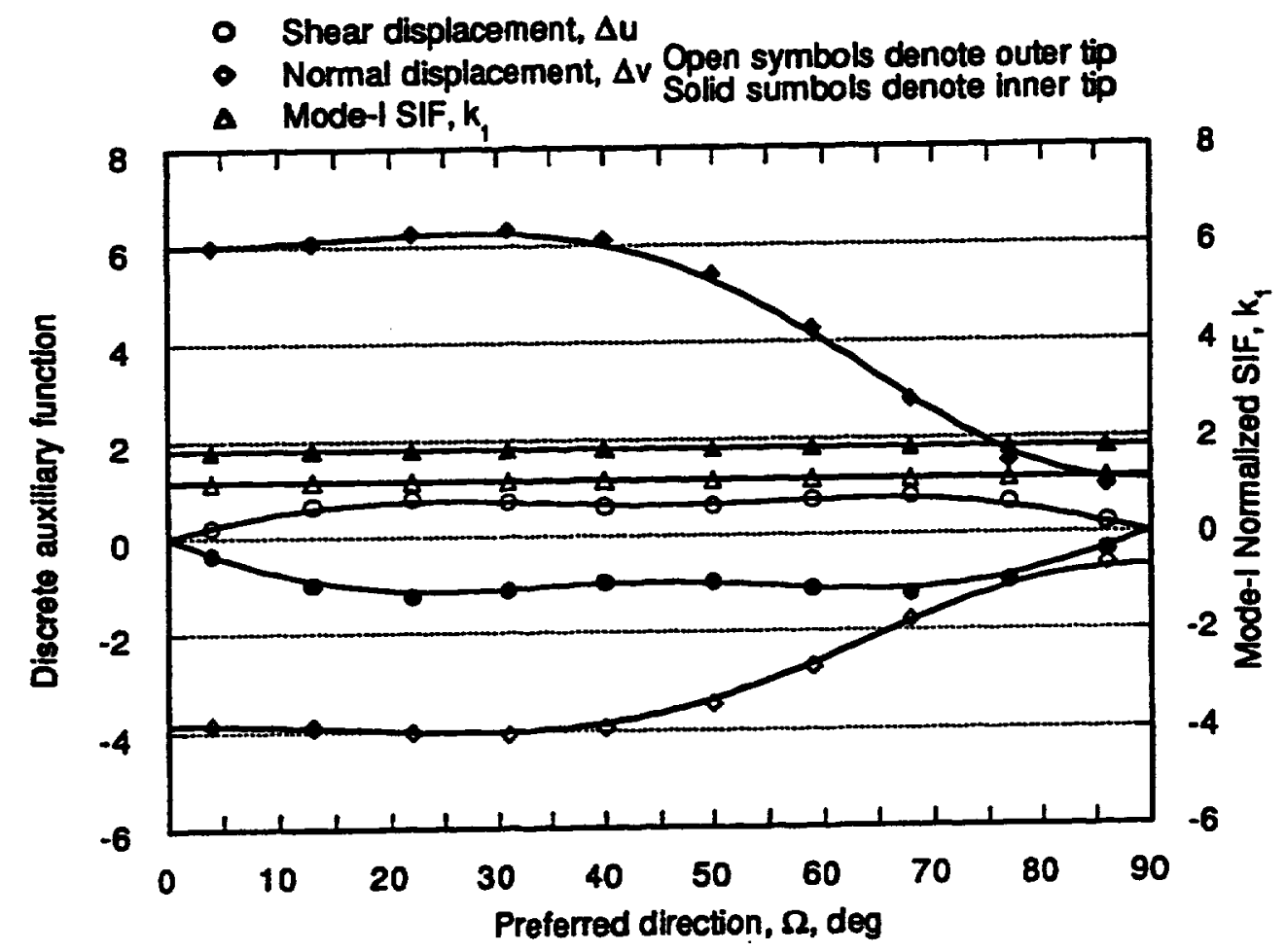

Figure 7: Discrete auxiliary functions and normalized SIF's versus $\Omega$ for two equal collinear cracks in a composite plate (strength of anisotropy $E_{L L} / E_{T T}=40$; subjected to far-field normal stress,see Fig. 3). 
shear displacements are zero only for the special cases in which the preferred direction is parallel $\left(\Omega=0^{\circ}\right)$ or normal $\left(\Omega=90^{\circ}\right)$ to the crack configuration; otherwise both openings have nonzero values. The local shear crack-opening displacements maxima occur at $\Omega=22^{\circ}$ and $68^{\circ}$, whereas the local nonzero minimum for the shear displacement occurs at $\Omega=45^{\circ}$. Conversely, the normal crack-opening displacements are approximately constant over the range $0<\Omega<40$ with the maximum value occurring at $\Omega=30^{\circ}$; it then significantly decreases when $\Omega>40$, until a value equivalent to $30 \%$ of the maximum normal opening is reached at $\Omega=90^{\circ}$. Clearly, this indicates that although mixed modedisplacements may be observed, in actuality, counter to common expectations resulting from isotropic materials, the anisotropic case may have only a mode-I driving force.

In Figure 8 the MSP's are shown as a function of the preferred direction orientation angle $\Omega$. Note that the $E_{x y}^{(1)}$ curve relative to the curve $E_{y y}^{(2)}$ is symmetrical about the line $\Omega=45^{\circ}$. The other two MSP's are again equal to each other and to zero for the orthogonal cases $\Omega=0^{\circ}$ and $90^{\circ}$. Consequently, $E_{x y}^{(1)}$ and $E_{y y}^{(2)}$ must be related to b $b_{16}$ and $b_{26}$ (see eq. (9)). In order to give a clearer physical interpretation to $E_{x y}^{(1)}$ and $E_{y y}^{(2)}$ let us relate them to the corresponding material parameters $E_{L L}$ and $E_{T T}$.

Considering the fact that

$$
E_{x y}^{(2)}=E_{y y}^{(1)}
$$

we can substitute eqs. (54) and (55) into eq. (101) and solve for $b_{22}$ :

$$
b_{22}=b_{11}\left(a^{2}+b^{2}\right)\left(c^{2}+d^{2}\right)
$$

Or in terms of the roots of the characteristic equation

$$
\mu_{1} \mu_{2} \mu_{3} \mu_{4}=\frac{b_{22}}{b_{11}}
$$

In the case of a transversely isotropic or orthotropic material, $b=d=0$, so eq. (103) reduces to a well-known mathematical relation of the following form:

$$
a^{2} c^{2}=\frac{E_{L L}}{E_{T T}}
$$

By substituting eq. (102) into eq. (56), and the resulting equation into eq. (53), we find that

$$
E_{x y}^{(1)}=E_{y y}^{(2)} \frac{c\left(a^{2}+b^{2}\right)+a\left(c^{2}+d^{2}\right)}{(a+c)}
$$

Thus, by using eq. (104) together with the transversely isotropic or orthotropic form of eq. (105) (i.e., $b=d=0$ ) we can show that

$$
\left[\frac{E_{x y}^{(1)}}{E_{y y}^{(2)}}\right]_{b=d=0}=a c=\sqrt{\frac{E_{L L}}{E_{T T}}}
$$

On the basis of eq. (106) and our observations from Fig. 8 we may conclude that for the general anisotropic case, $E_{x y}^{(1)}$ is proportional to the square root of the effective Young's modulus in the local $x$-direction and $E_{y y}^{(2)}$ is proportional to the square root of the effective Young's modulus in the local $y$-direction. Note, however, for the general anisotropic case the constants of proportionality are interdependent. 


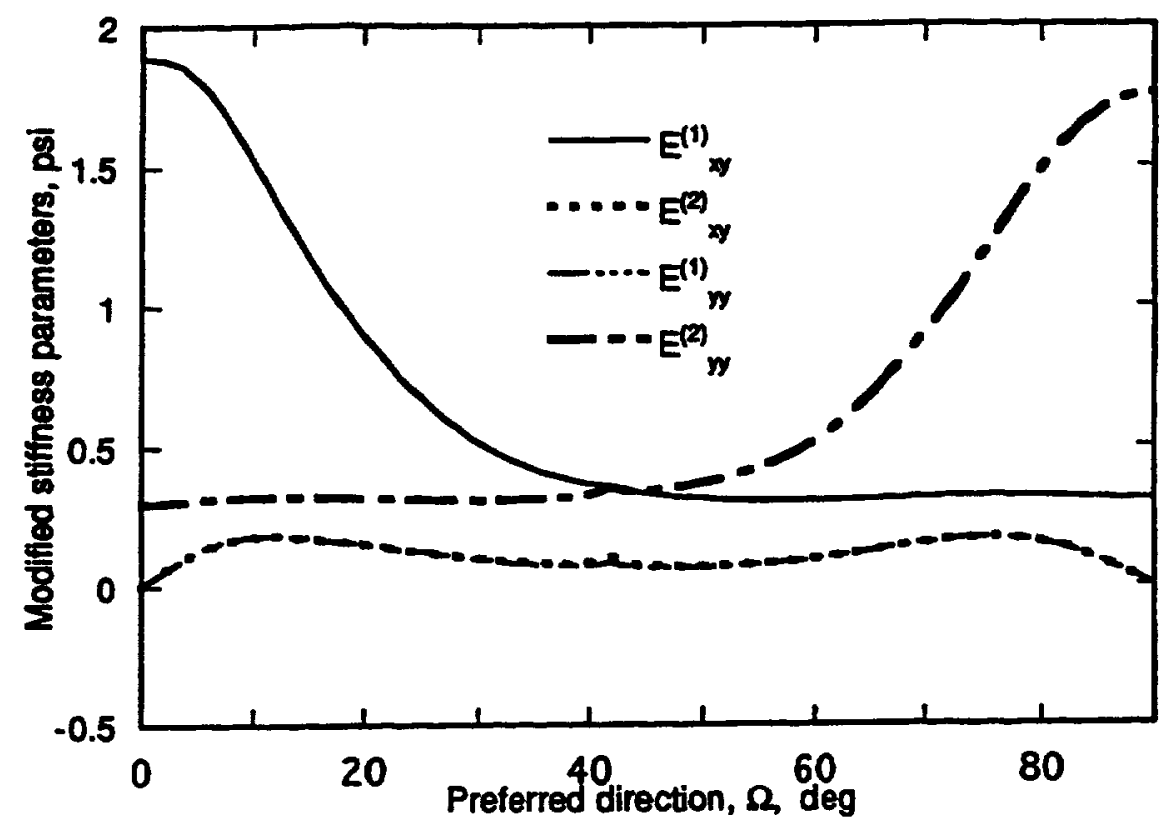

Figure 8: Modified stiffness parameters versus $\Omega$ for two equal collinear cracks in a composite plate (strength of anisotropy $E_{L L} / E_{T T}=40$; subjected to far-field normal stress, see Fig. 3).

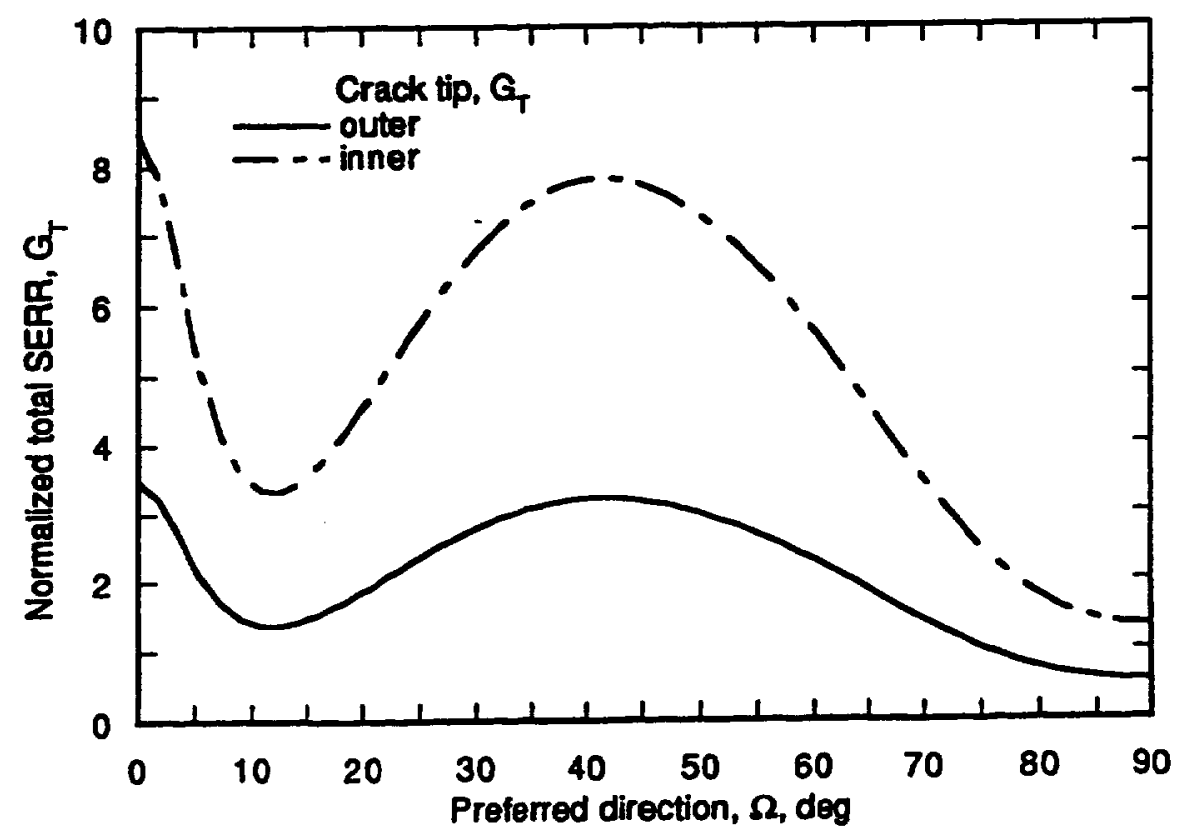

Figure 9: Normalized $G_{T}$ versus $\Omega$ for two equal collinear cracks in a composite plate (strength of anisotropy $E_{L L} / E_{T T}=40$; subjected to far-field normal stress, see Fig. 3). 
The importance of being able to use the $G_{T}$ as a damage propagation criterion is once again illustrated in Fig. 9, which shows the total $G_{T}$ versus the orientation angle $\Omega$. Clearly, there are a number of angles at which $\mathrm{G}_{T}$ extrems exist. The global maximum occurs at $\Omega=0^{\circ}$, and the global minimum at $\Omega=90^{\circ}$, for both inner and outer crack tips. The other two important angles at which the $G_{T}$ 's reach a local minimum and maximum are $\Omega=12^{\circ}$ and $\Omega=42^{\circ}$, respectively. Again a comparison of Figs. 7 and 9 shows that the $G_{T}$ 's can indicate the critical angles of interest, whereas the SIF's are completely insensitive to variation of the preferred direction.

\subsubsection{Parallel cracks}

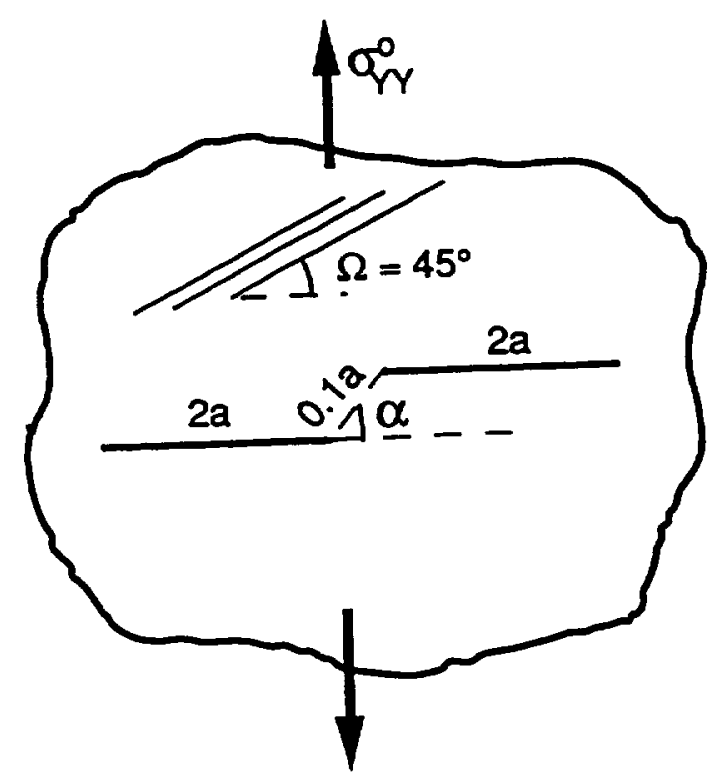

Figure 10: Geometry and loading condition defining two parallel crack problem.

Now let us consider the variation of the SIF's, the crack-opening displacements, and the $\mathrm{G}_{T}$ 's for the case when the two cracks are not collinear. A convenient parameter that can be controlled is the inclination angle designated $\alpha$ between the horizontal axis and the line connecting the inner crack tips in Fig. 10. An example of the previous case (collinear cracks) is easily obtained by setting $\alpha=0^{\circ}$. The constant parameters are the distance between inner crack tips, $0.1 a_{1}$ (where $2 a_{1}$ is the crack length of crack number 1), the strength of anisotropy $E_{L L} / E_{T T}=40$; and the preferred direction $\Omega=45^{\circ}$. As a result of changing the crack configuration, the SIF's are no longer constant for the inner crack tips; those associated with the outer crack tips, however, are not significantly influenced. As illustrated in Fig. 11, mode-I SIF for the inner crack tip monotonically increases with the angle $\alpha$; whereas mode-II SIF is zero only for $\alpha=0^{\circ}$ and $90^{\circ}$ and attains a maximum at $\alpha=\Omega=45^{\circ}$, as we might expect.

It is interesting to analyze the discrete auxiliary functions which approximate the shear and normal crack-opening displacements, $\Delta u$ and $\Delta v$. The shear term of the inner 


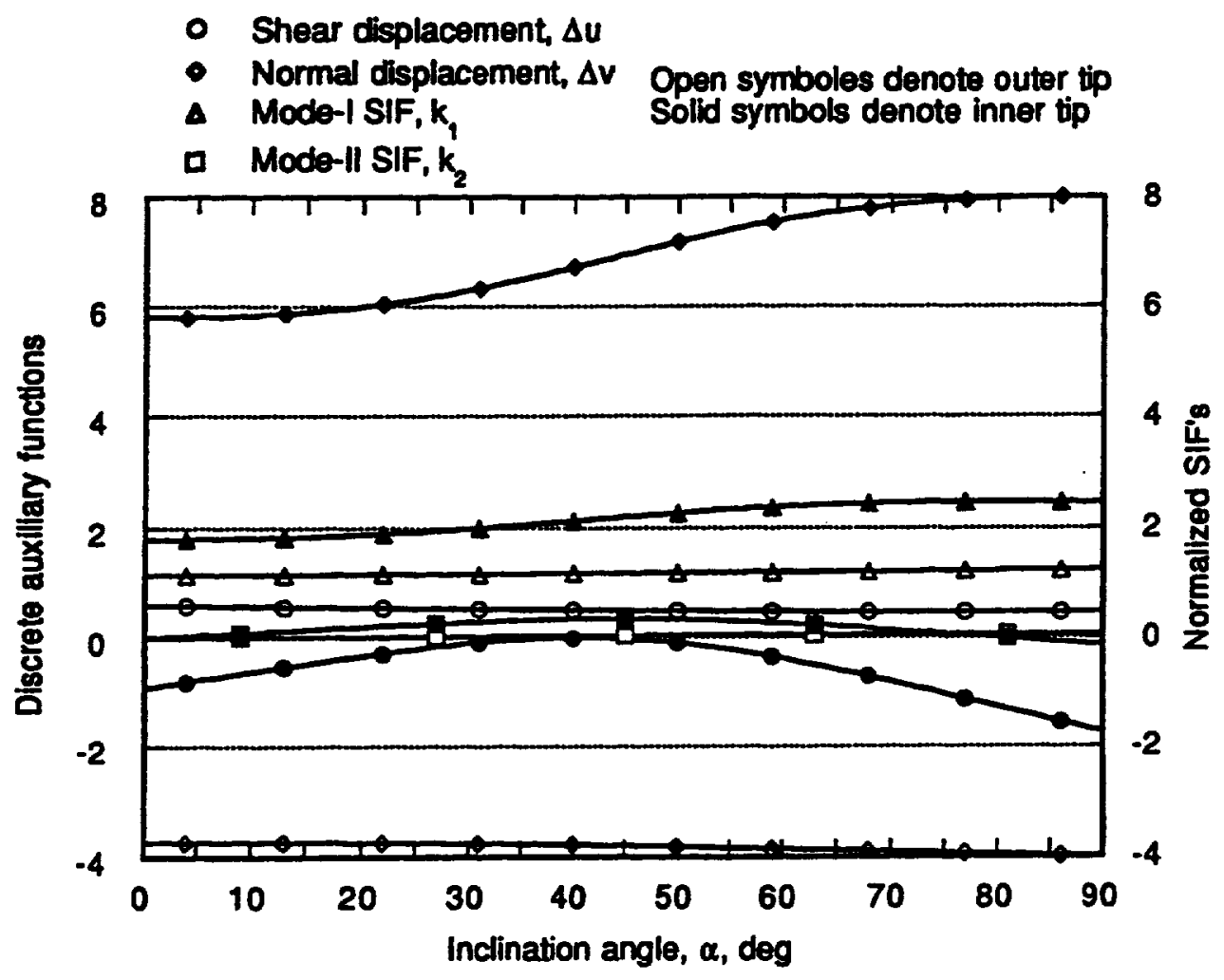

Figure 11: Discrete auxiliary functions and normalized SIF's versus $\alpha$ for two equal noncollinear cracks in a composite plate ( strength of anisotropy $E_{L L} / E_{T T}=40$; reinforced at $\Omega=45^{\circ}$; subjected to far-field normal stress, see Fig. 10 ). 
crack tip is zero only when $\alpha=40^{\circ}$ (even though the shear stress is at a maximum). It is larger for this configuration at $\alpha=90^{\circ}$ (when the shear stress is zero) than it is for the collinear crack configuration. Normal crack-opening displacements are the largest also for $\alpha=90^{\circ}$. Note that changing the crack configuration by changing $\alpha$ does not change the material properties because they are defined in the local coordinate system; therefore, given the preferred direction $\Omega=45^{\circ}$ we can find the pertinent MSP's in Fig. 8.

Once again the combined effect of mode-I and -II SIF's and the normal and shear local displacements are captured by the single scalar measure known as the $G_{T}$ (see eq. (97)). Figure 12 shows the $G_{T}$ at both the inner and outer crack tips as a function of the crack configuration angle $\alpha$. The shapes of these curves resemble the shapes of $k_{1}$ and $k_{2}$ for the inner crack tips (the degree of influence that $k_{1}$ and $k_{2}$ have on the $G_{T}$ is clearly shown in eq. (99)). Since the maximum values of $k_{1}$ and $k_{2}$ are reached at $90^{\circ}$ and $45^{\circ}$, respectively, the maximum value of the $G_{T}$ would be expect to occur somewhere in between. Figure 12 indicates that for the inner crack tips this maximum is reached when $\alpha=70^{\circ}$; however, at the outer crack tip the $G_{T}$ is only slightly affected (as are $k_{1}$ and $k_{2}$ ) by a change in $\alpha$, and it reaches its maximum when $\alpha=90^{\circ}$.

\subsubsection{Nonparallel Cracks}

Consider a related case wherein the material strength of anisotropy is $E_{L L} / E_{T T}=40$, the preferred direction is denoted by $\Omega=30^{\circ}$, and the two cracks of equal length are configured as shown in the Fig. 13. This figure indicates that crack $a b$ remains horizontal, while crack $c d$ rotates around the crack tip $c$; where the crack tip distance between tips $b$ and $c$ remains constant. Here we will examine the influence of varying the angular orientation of crack $c d, \phi(2)$, from 0 to $180^{\circ}$. Figures 14 and 15 show the mode-I and $-I I$ SIF's, respectively, at the four crack tips. Notice that the outer crack tip of the horizontal crack (tip $a$ ) is only slightly affected by the change in angular orientation of the crack $c d$. Whereas, the other crack tips $(b, c$, and $d)$ display significant and complex interactive behavior for both mode-I and -II SIF. For example, mode-I SIF for crack tip b has a local maximum at 21,42 and $132^{\circ}$, and a local minimum at $0,31,122$, and $180^{\circ}$, while crack tip $c$ starts at the same value as tip $b$, then smoothly decreases to approximately zero (within the range $80<\phi(2)<140^{\circ}$ ) whereupon the SIF sharply rises to again the same value of SIF as that of crack tip $a$, at $\phi(2)=180^{\circ}$. Similarly, extremum are observed for the mode-II SIF at a variety of angle, that is $20,43,110,120$ and $132^{\circ}$.

The actual calculated $\mathrm{G}_{T}$ 's are shown in Fig. 16. By comparing Fig.16 to Figs. 14 and 15, we can observe that the location of the extremum for the $G_{T}$ 's are similar to the SIF's. However, it is evident that the $G_{T}$ for outer crack tips $a$ and $d$ are dominated by mode-I SIF, whereas inner crack tips $b$ and $c$ are significantly influenced by both modes. Figure 16 also shows that $G_{T}$ is the largest at crack tip $b$ for $0^{\circ}<\phi(2)<100^{\circ}$ and $123^{\circ}<\phi(2)<153^{\circ}$ indicating possible self similar crack propagation within this range. Note that when crack $c d$ becomes aligned with the preferred fiber direction, $\mathrm{G}_{T}$ (at crack tip b) reaches a minimum while crack tip $c$ reaches a maximum. Thus, we may conclude that at this critical configuration, $\phi(2)=30^{\circ}$, the inner crack tips $b$ and $c$ may be driven towards each other and connect to form a macro kinked crack. 


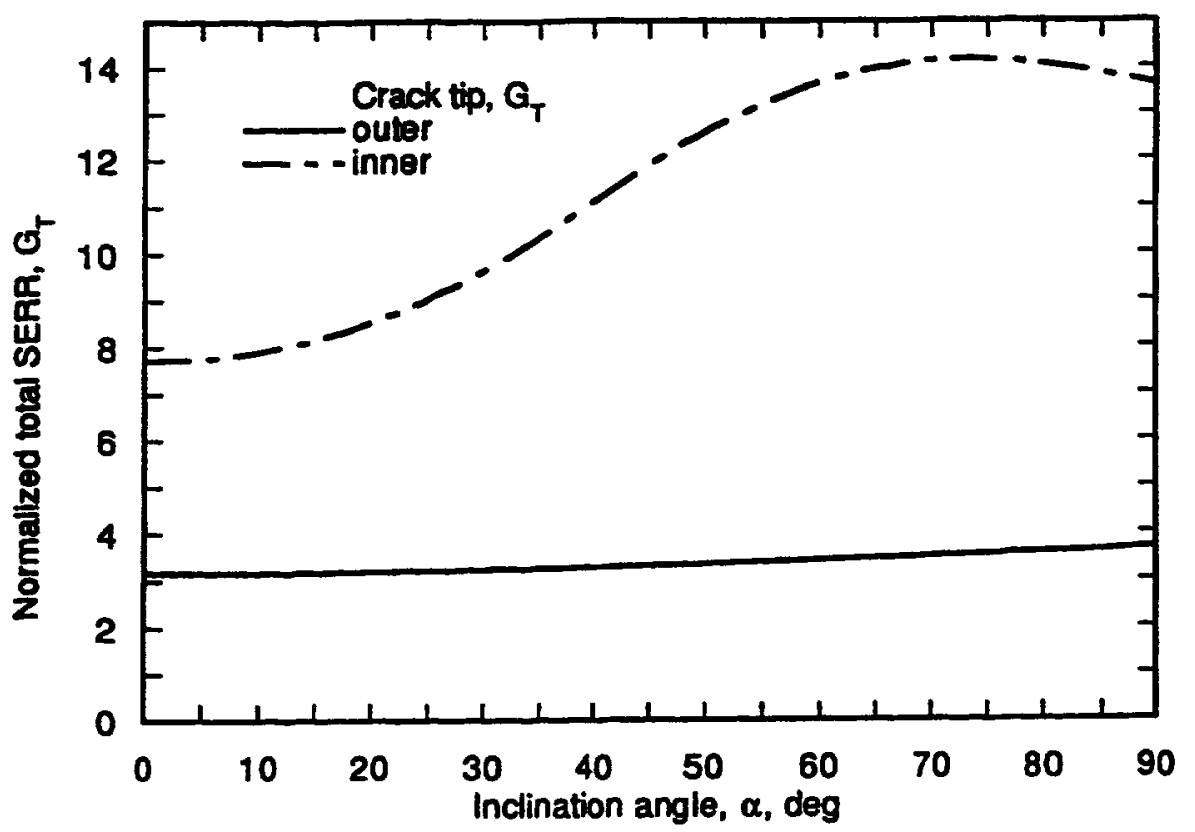

Figure 12: Normalized $G_{T}$ versus $\alpha$ for two equal noncollinear cracks in a composite plate (strength of anisotropy $E_{L L} / E_{T T}=40$; reinforced at $\Omega=45^{\circ}$; subjected to far-field normal stress, see Fig. 10).

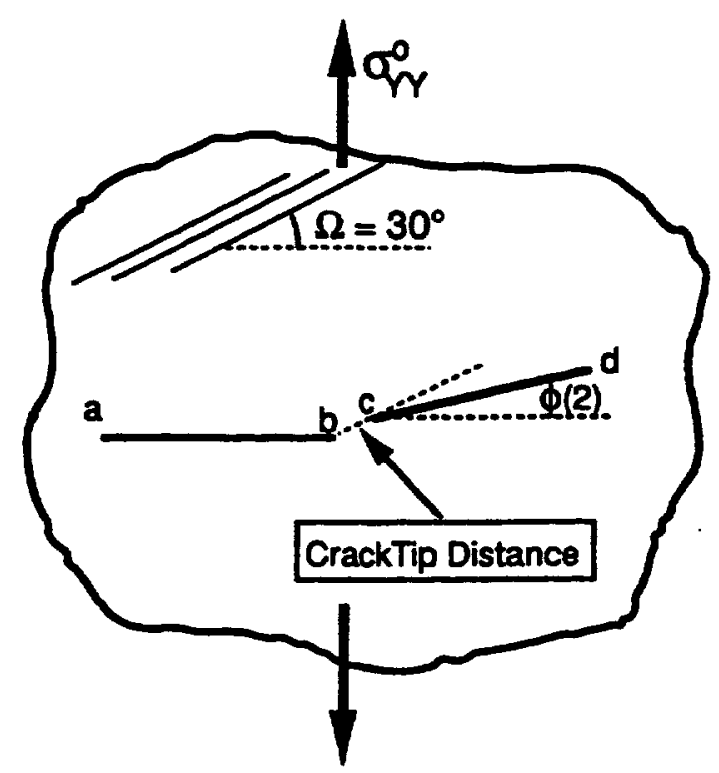

Figure 13: Geometry and loading condition problem of two non-parallel cracks. 


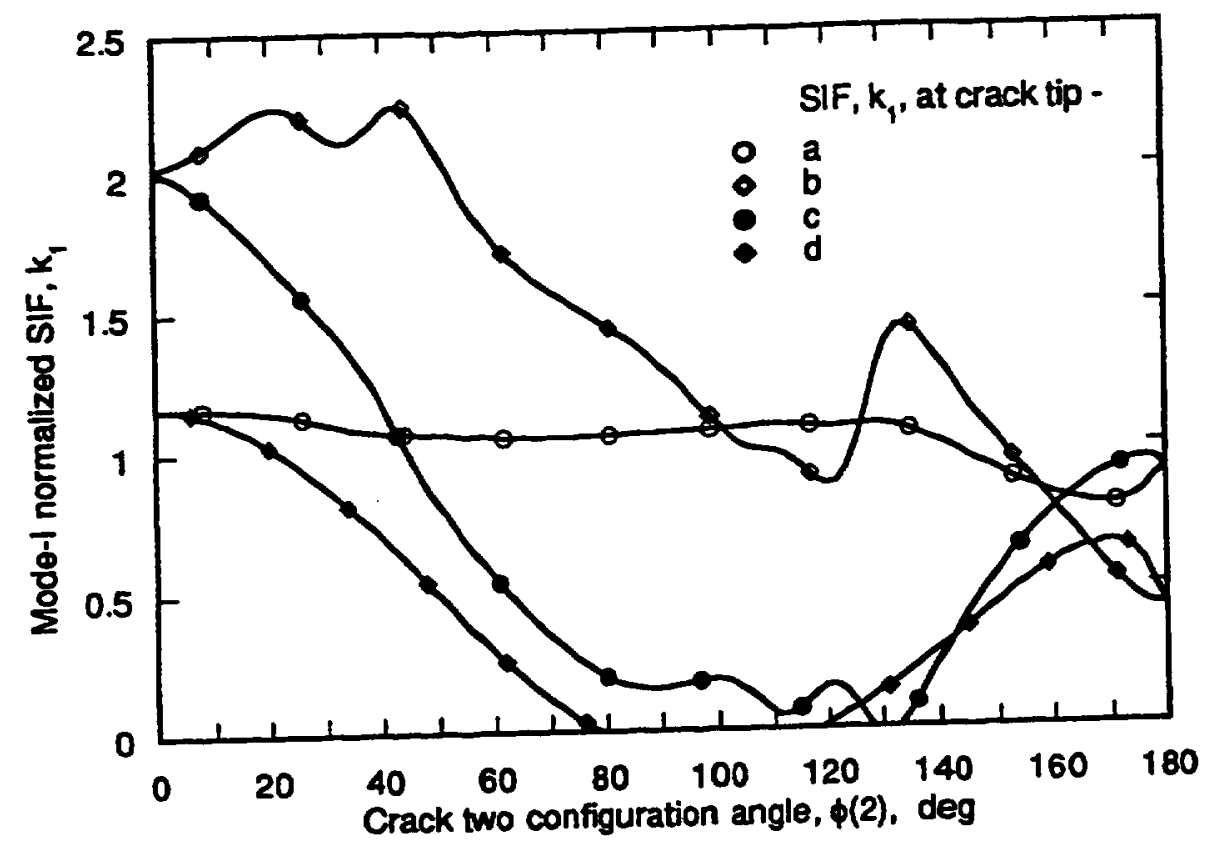

Figure 14: Normalized mode-I SIF's versus crack-2 angle $\phi(2)$ for two equal cracks in a composite plate (strength of anisotropy $E_{L L} / E_{T T}=40$; reinforced at $\Omega=30^{\circ}$; subjected to far-field normal stress, see Fig. 13).

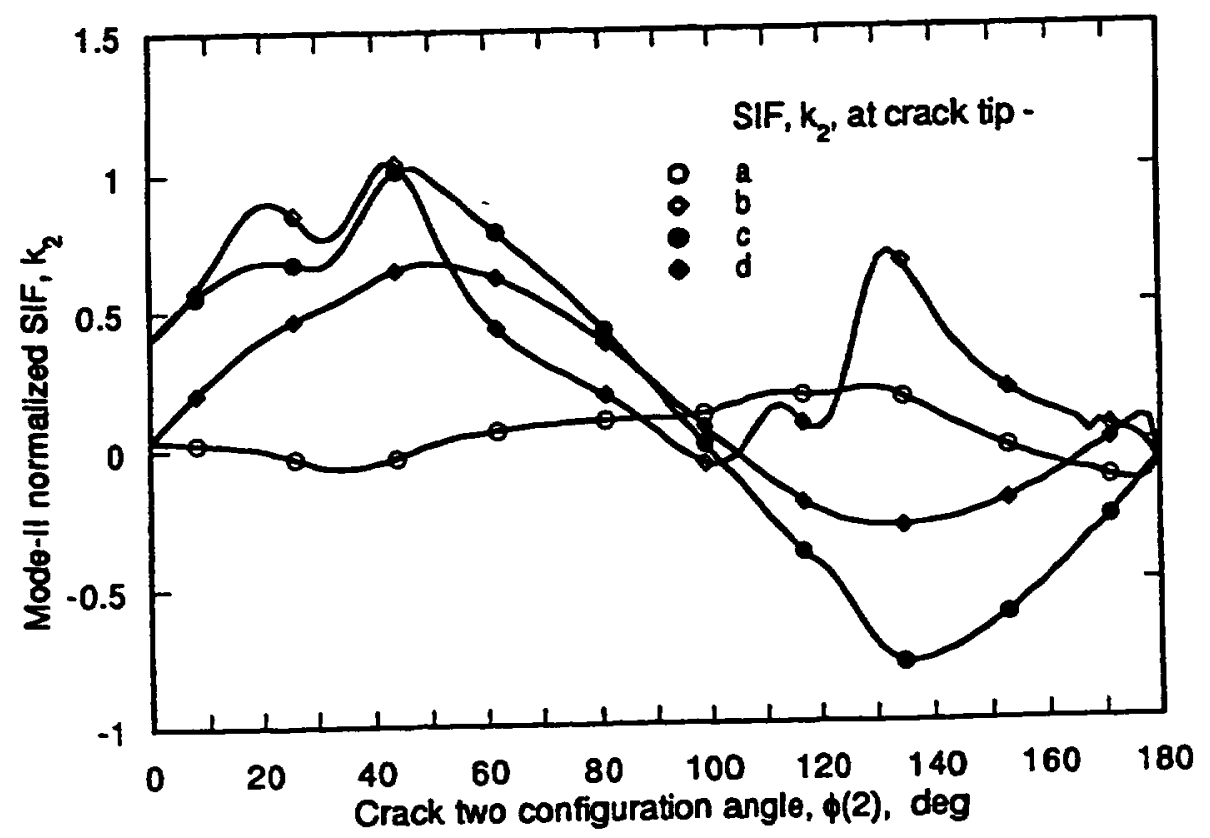

Figure 15: Normalized mode-II SIF's versus crack-2 angle $\phi(2)$ for two equal cracks in a composite plate (strength of anisotropy $E_{L L} / E_{T T}=40$; reinforced at $\Omega=30^{\circ}$;subjected to far-field normal stress, see Fig. 13). 


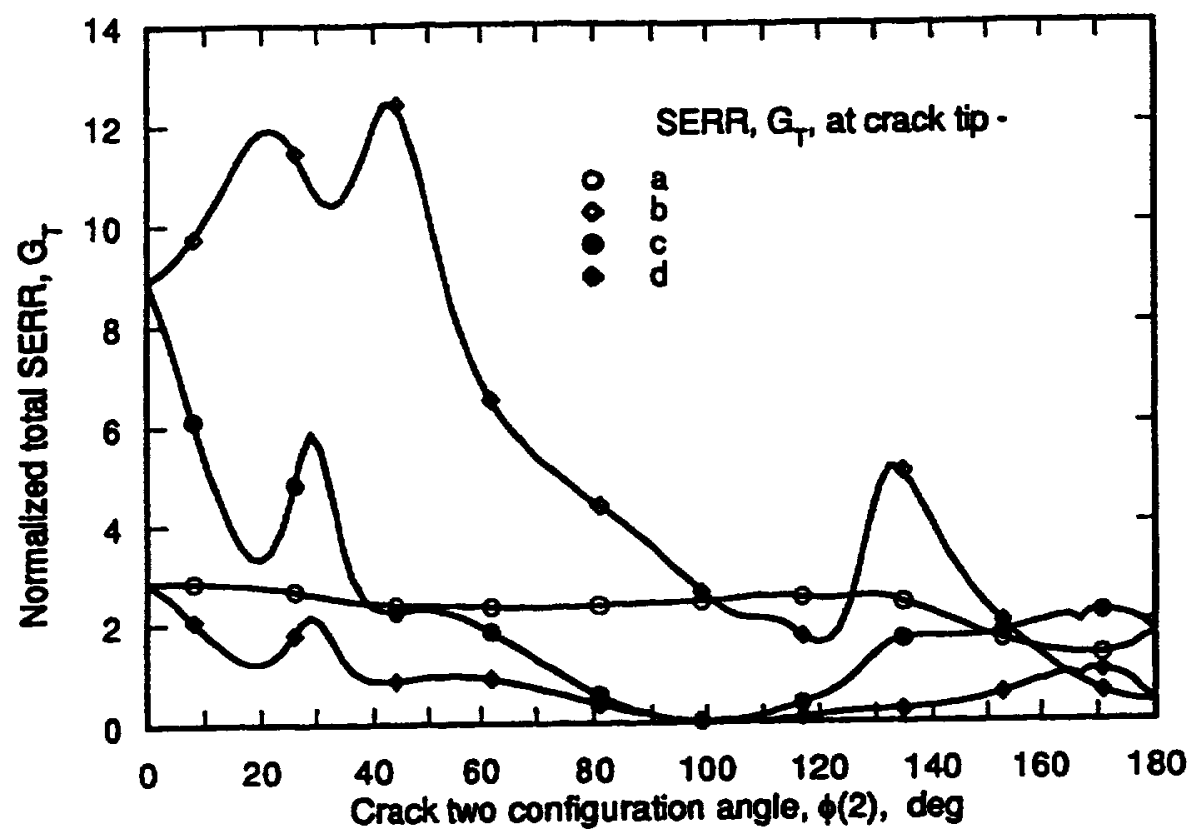

Figure 16: Normalized $G_{T}$ versus crack-2 angle $\phi(2)$ for two equal cracks in a composite plate (strength of anisotropy $E_{L L} / E_{T T}=40$; reinforced at $\Omega=30^{\circ}$; subjected to far-field normal stress, see Fig 13.

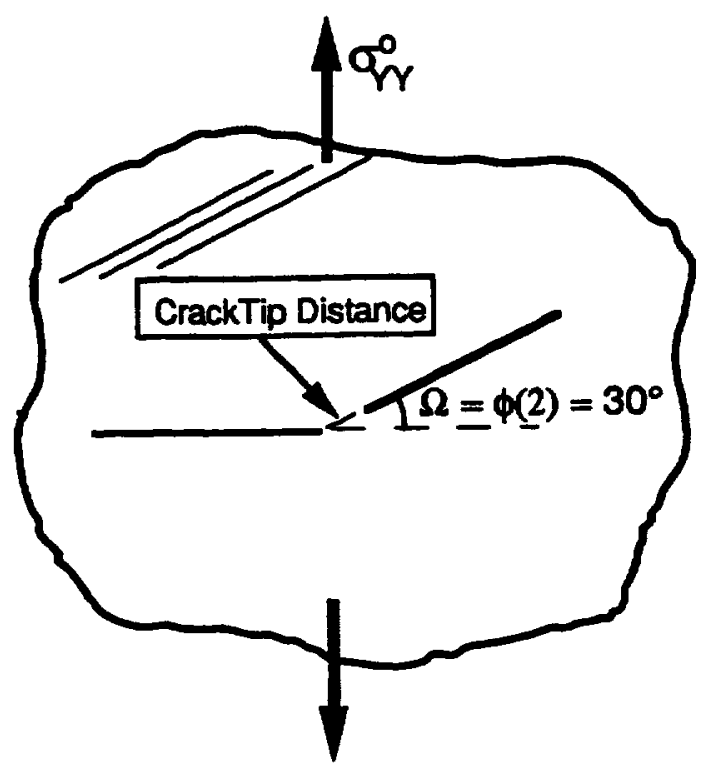

Figure 17: Geometry and loading condition of two inclined cracks. 


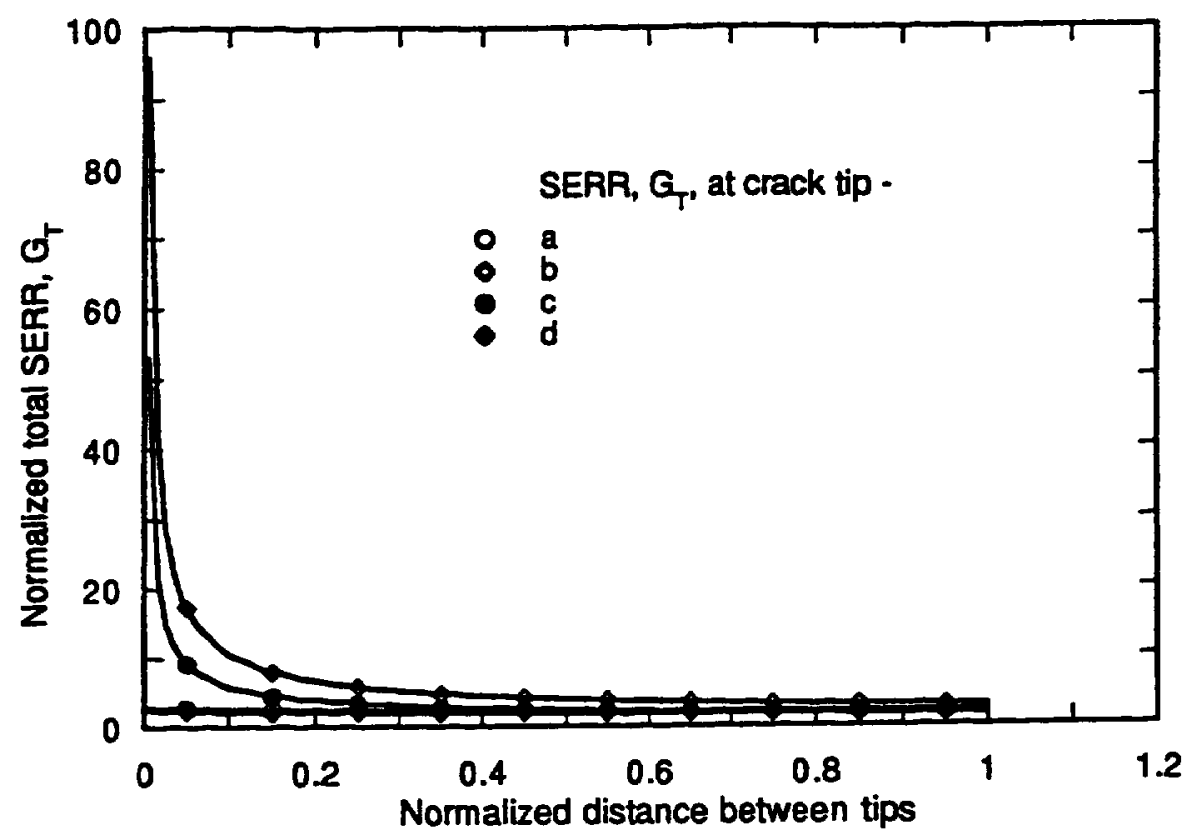

Figure 18: Normalized $\mathrm{G}_{\boldsymbol{T}}$ versus normalized crack tip distance for inclined cracks in a composite plate (strength of anisotropy $E_{L L} / E_{T T}=40 ;$ reinforced at $\Omega=30^{\circ}$; subjected to far-field normal stress, see Fig. 17).

\subsubsection{Influence of Crack Spacing}

To determine the influence of crack spacing, let us consider the preceding two crack system for the critical configuration $\phi(2)=\Omega=30^{\circ}$ and analyze the behavior of these cracks to examine how the $G_{T}$ varies with respect to the normalized inner crack tip distance $d$ (see Fig. 17). As we would expect, given the results shown in Fig. 13, the $G_{T}$ 's of the outer crack tips are hardly influenced by the change in positions of the cracks. But the $G_{T}$ 's for the inner crack tips ( $b$ and $c$ ) display a strong interaction, especially for $0<d<0.2 a_{1}$, as shown in Fig. 18: the closer the crack tips, the higher the normalized $G_{T}$, and thus, the smaller the far-field stress state required to cause the cracks to propagate toward each other.

\subsection{Three Crack Interaction}

Consider a transversely isotropic plate with a strength of anisotropy $E_{L L} / E_{T T}=40$ and a three-parallel-crack system as shown in Fig. 19. The two cracks denoted ef and od are always symmetric, with respect to the horizontal line that coincides with crack $a b$. First let us keep the distance between inner crack tips $b, c$, and $e\left(D_{h}\right)$ constant at $0.1 a_{1}$, while the angle $\Omega$, describing the preferred direction, is varied. Mode-I SIF's for tips $b, c$, and $e$ are shown in Fig. 20. A number of observations can be made from Fig. 20. First, the $k_{1}$ for crack tip $b$ resembles an inverted parabola with a maximum at $\Omega=45^{\circ}$. Second, $k_{1}$ for crack tip $b$ is the largest of the three inner tips - for all preferred directions because of the magnification influence of cracks $e f$ and $c d$ (situated 


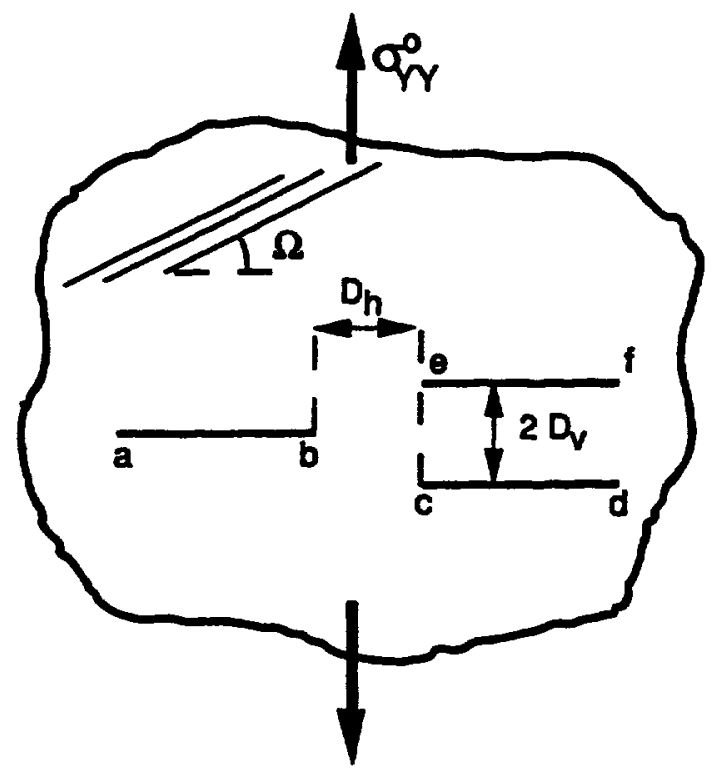

Figure 19: Geometry and loading condition defining three parallel cracks problem.

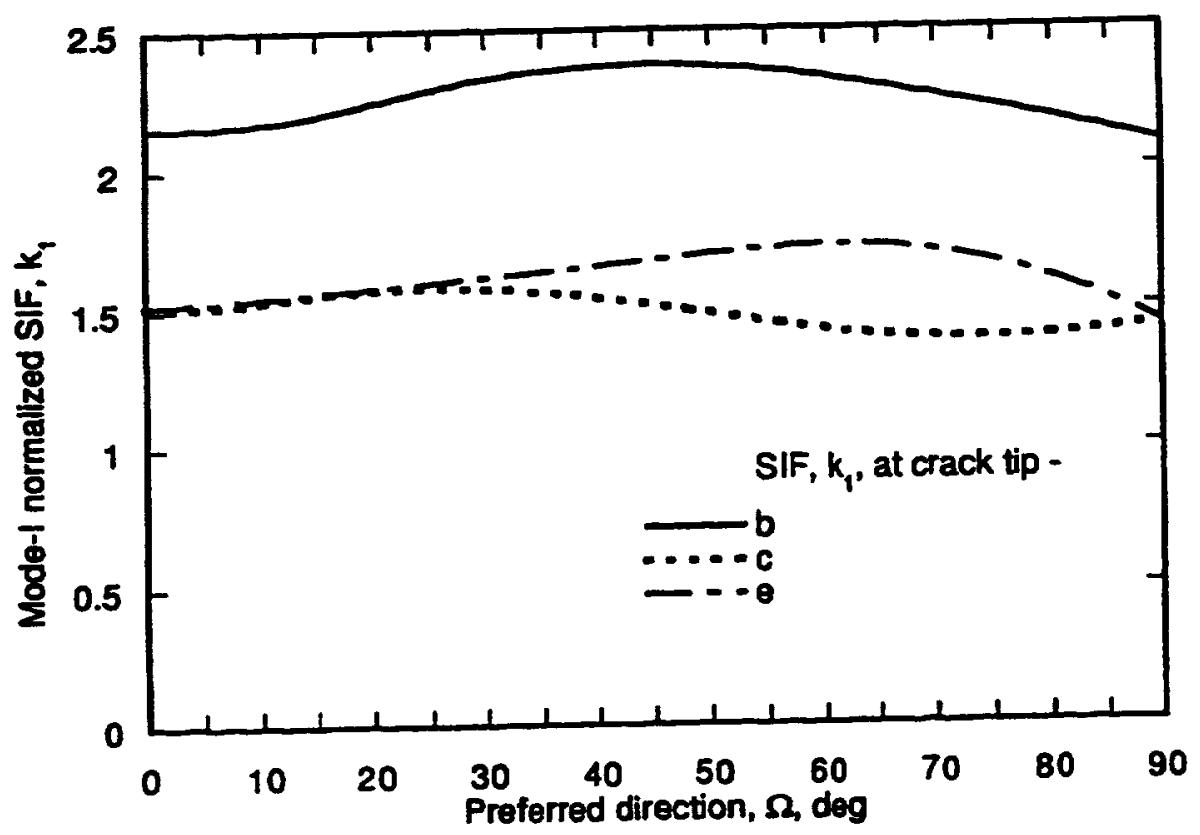

Figure 20: Mode-I normalized SIF's versus $\Omega$ for three parallel cracks in a composite plate (strength of anisotropy $E_{L L} / E_{T T}=40$; subjected to far-field normal stress, see Fig. 19). 


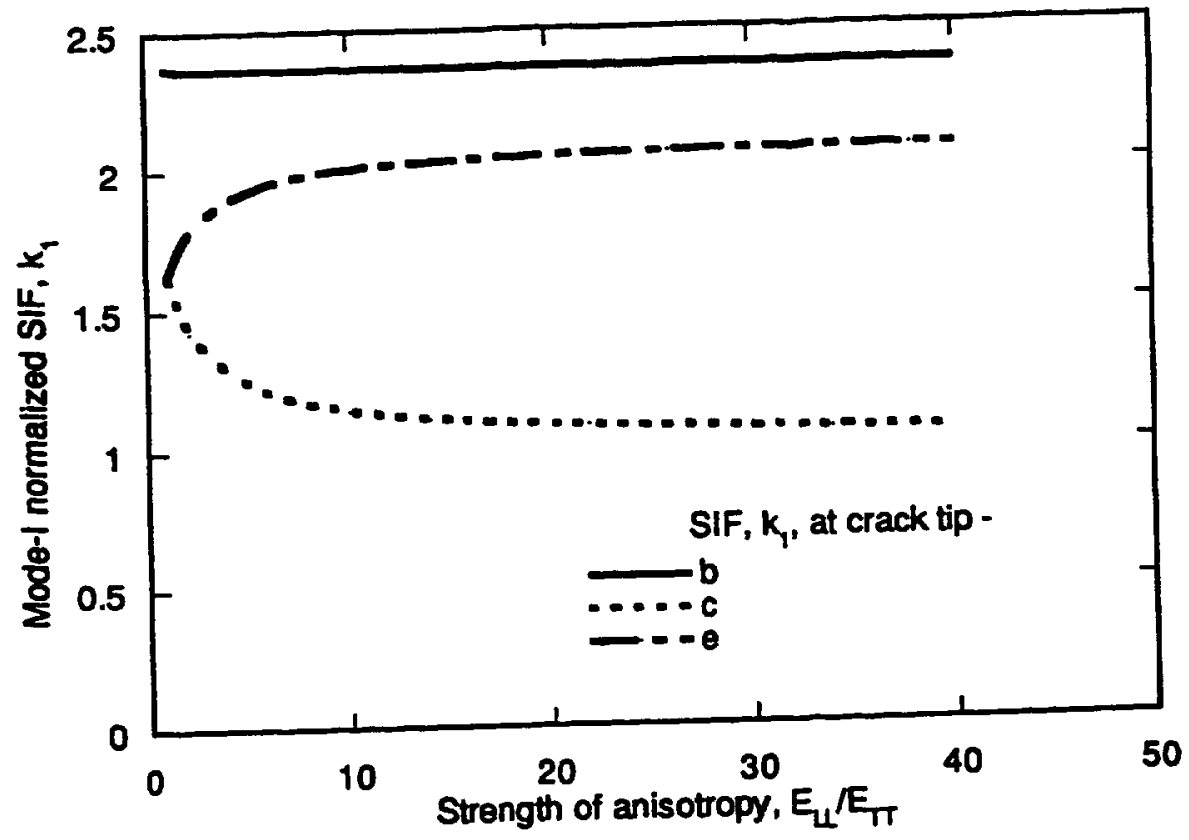

Figure 21: Mode-I normalized SIF's versus strength of anisotropy $E_{L L} / E_{T T}$ for three parallel cracks in a composite plate (reinforced at $\Omega=60^{\circ}$; subjected to far-field normal stress, see Fig. 19).

in front of crack $a b$ ). Similarly, because of the mutual shielding of the cracks above and below, the $k_{1}$ for cracks $c d$ and $e f$ must be smaller. Third, the $k_{1}$ at inner tips $c$ and $e$ are identical in the range $0<\Omega<25$, but they begin to deviate from one another, in a symmetrical manner, for preferred directions $\Omega>25^{\circ}$. Also since $k_{1}$ for crack tip $e$ is larger than that for crack tip $c$, this would suggest that the influence of the singular stresses is transmitted over greater distances along the preferred direction, thus confirming the concept of stress-channeling (or in our case damage channeling) along the preferred direction, as discussed by Spencer[12]. The distance of influence is clearly dependent on the strength of anisotropy specified, as can be seen in Fig. 21.

Mode-II SIF's for the three inner crack tips are shown in Fig. 22. Here, the absolute value of $k_{2}$ for crack tip $b$ is approximately zero for the various preferred directions and exactly zero for the orthogonal conditions $\left(\Omega=0,90^{\circ}\right)$. The absolute value of $k_{2}$ for crack tip $e$ is the largest of the three with a local maximum at $\Omega=25^{\circ}$. Again, this is a function of the directional stress channeling effect. Also, note that the absolute values of $k_{2}$ for crack tips $c$ and $e$ are identical when the cracks are parallel and normal to the preferred directions; however the magnitude at $\Omega=0$ is more than twice that for $\Omega=90$.

The variation of the $G_{T}$ as a function of preferred direction, for the three inner crack tips $b, c$, and $e$ are shown in Fig. 23. Clearly, the $\mathrm{G}_{T}$ combines all of the aforementioned characteristics for each crack tip into one convenient parameter that exhibits a strong dependence on the preferred direction angle $\Omega$. Local maxima for these curves are located within a $10^{\circ}$ range centered at $\Omega=45^{\circ}$. It may be concluded from Fig. 23 that crack propagation is easiest when the preferred direction makes a $45^{\circ}$ angle, thus connecting through reinforcement (or damage channeling) crack tips $b$ and $e$. Hence, as a result of 


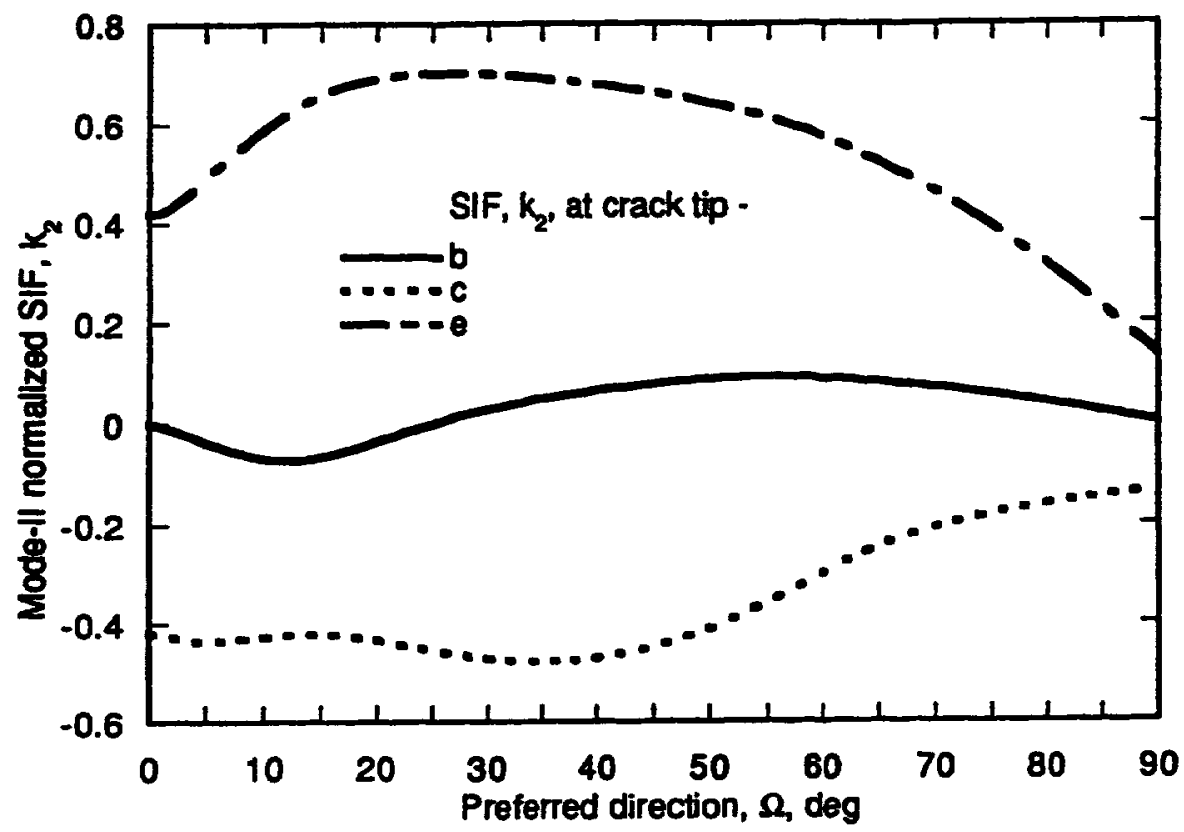

Figure 22: Mode-II normalized SIF's versus angle $\Omega$ for three parallel cracks in a composite plate (strength of anisotropy $E_{L L} / E_{T T}=40$; subjected to far-field normal stress, see Fig. 19).

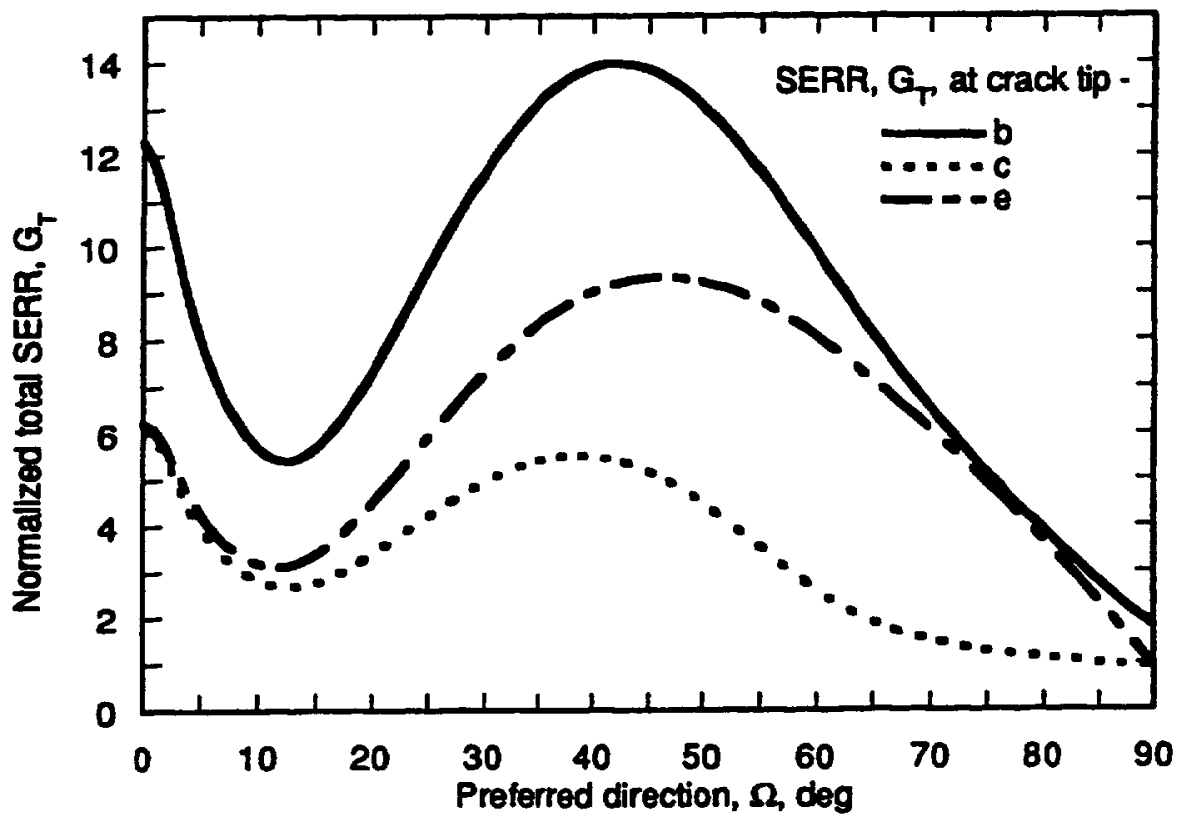

Figure 23: Normalized $G_{T}$ versus $\Omega$ for three parallel cracks in a composite plate (strength of anisotropy $E_{L L} / E_{T T}=40$; subjected to far-field normal stress, see Fig. 19). 


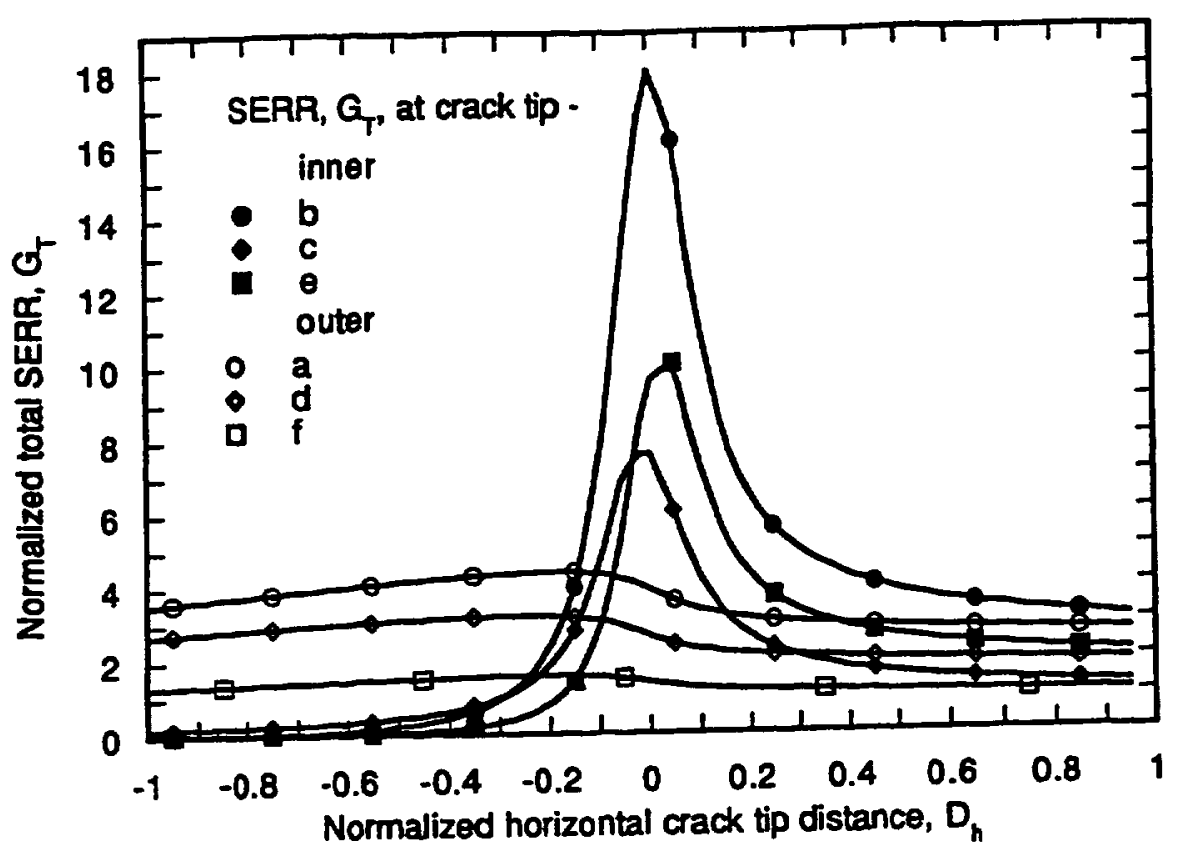

Figure 24: Normalized $G_{T}$ versus horizontal component of the tip distance $D_{h}$ for three parallel cracks in a composite plate (strength of anisotropy $E_{L L} / E_{T T}=40$; reinforced at $\Omega=45^{\circ}$; subjected to far-field normal stress, see Fig. 19).

a physical crack propagation, we would anticipate a zigzag crack that develops through the connection of cracks $a b$ and $e f$.

Last, let us consider the case of two parallel cracks ef and $c d$ that are a fixed vertical distance apart $\left(D_{v}=0.1 a_{1}\right)$ and crack $a b$ is slid in between them. Figure 24 shows the variation of the $G_{T}$ 's for all crack tips as a function of horizontal position $D_{h}$ for the case when $\Omega=45^{\circ}$. When the parameter $D_{h}$ is zero, tip $b$ is on the same vertical line that connects tips $c$ and $e$. Thus, when crack $a b$ is away from the parallel cracks $D_{h}>0$, and when tip $b$ is between cracks $c d$ and $e f, D_{h}<0$. Note that as crack tip $b$ comes closer to the vertical line connecting tips $c$ and $e$, the $G_{T} s$ are magnified; with the amplification factor of the inner crack tips being significantly greater than that of the outer crack tips. Conversely, when $D_{h}<0$, all inner tips become strongly shielded, so the inner $\mathrm{G}_{T}$ 's sharply drop off, almost to zero. Note that crack tip $e$ has the most rapid decrease and quickly reaches a value less than that of tip $c$.

\subsection{Horizontal Notch Interaction With Three Microcracks}

The final problem to be consider in this paper consist of a transversely isotropic plate, with a strength of anisotropy $\left(E_{L L} / E_{T T}\right)$ equal to 40 , that contains a large horizontal notch (of length $2 a_{1}$ ) and three radially oriented microcracks (of length $a_{2}=a_{3}=a_{4}=$ $0.1 a_{1}$ ), as shown in Fig. 25. The radial distance between the inner tips of the notch and the three microcracks remains fixed at $0.005 a_{1}$, while the orientation (angle $\Omega$ ) of the preferred direction is varied from 0 to $180^{\circ}$. The $G_{T}$ 's for all the inner microcrack and 


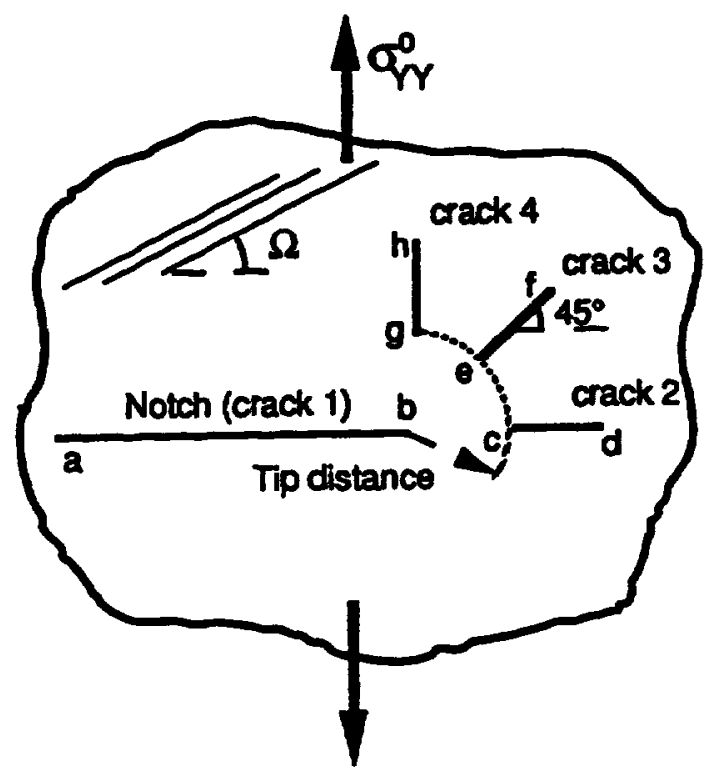

Figure 25: Geometry and loading condition defined for a horizontal notch and three microcrack problem.

notch tips are shown in Fig. 26.

Evidently, the $G_{T}$ for notch tip $b$ is larger than that for any of the three microcracks except when the orientation is within two small regions: $8^{\circ}<\Omega<20^{\circ}$ and $70^{\circ}<\Omega<98^{\circ}$. At these values the $G_{T}$ of the inner tip of the $45^{\circ}$ microcrack $e f$ becomes greater than that of the notch. All $G_{T}$ curves display strong and complex dependence on the orientation. Numerous local extrema exist with the maxima being noted as follows: for notch tip $b$, the local maxima occur at 0,38 , and $140^{\circ}$; for microcrack tip $g$, a maximum occurs at $30^{\circ}$; for microcrack tip $e$, maxima occur at 15,80 , and $155^{\circ}$; and for the horizontal microcrack tip $c$, the maxima occur at $0^{\circ}, 40^{\circ}$, and $150^{\circ}$. Again, if the maximum- $G_{T}$ criterion is used to predict crack propagation, we may conclude that the crack will kink by connecting with crack ef for the preferred direction when $8^{\circ}<\Omega<20^{\circ}$ and $70^{\circ}<\Omega<98^{\circ}$; otherwise it will propagate in a self-similar manner.

Now let us orient the preferred direction $\Omega$ at a fixed angle of $15^{\circ}$ and vary the radial inner tip distance between notch tip $b$ and the three microcrack tips $c, e$, and $g$. Figure 27 shows the amplification effect (which becomes noticeable when the tip distance is less then $0.1 a_{1}$ ) resulting from the complex interaction of the cloud of microcracks with the larger notch crack. Clearly, the $G_{T}$ for notch tip $b$ is the largest until the tip distance is decreased to approximately $0.04 a_{1}$, whereupon the $G_{T}$ for the microcrack inclined at $45^{\circ}$ drastically increases - exceeding all other curves and creating the condition for the crack to kink.

Finally, let us discuss the influence of the strength of anisotropy, as shown in Fig. 28. Here, we assume that the preferred direction and radial tip distance are held constant at $\Omega=15^{\circ}$ and $0.005 a_{1}$, respectively, while the strength of anisotropy $E_{L L} / E_{T T}$ is varied. When the material is isotropic (i.e., $E_{L L} / E_{T T}=1$ ) the $G_{T}$ at notch tip $b$ far surpasses 


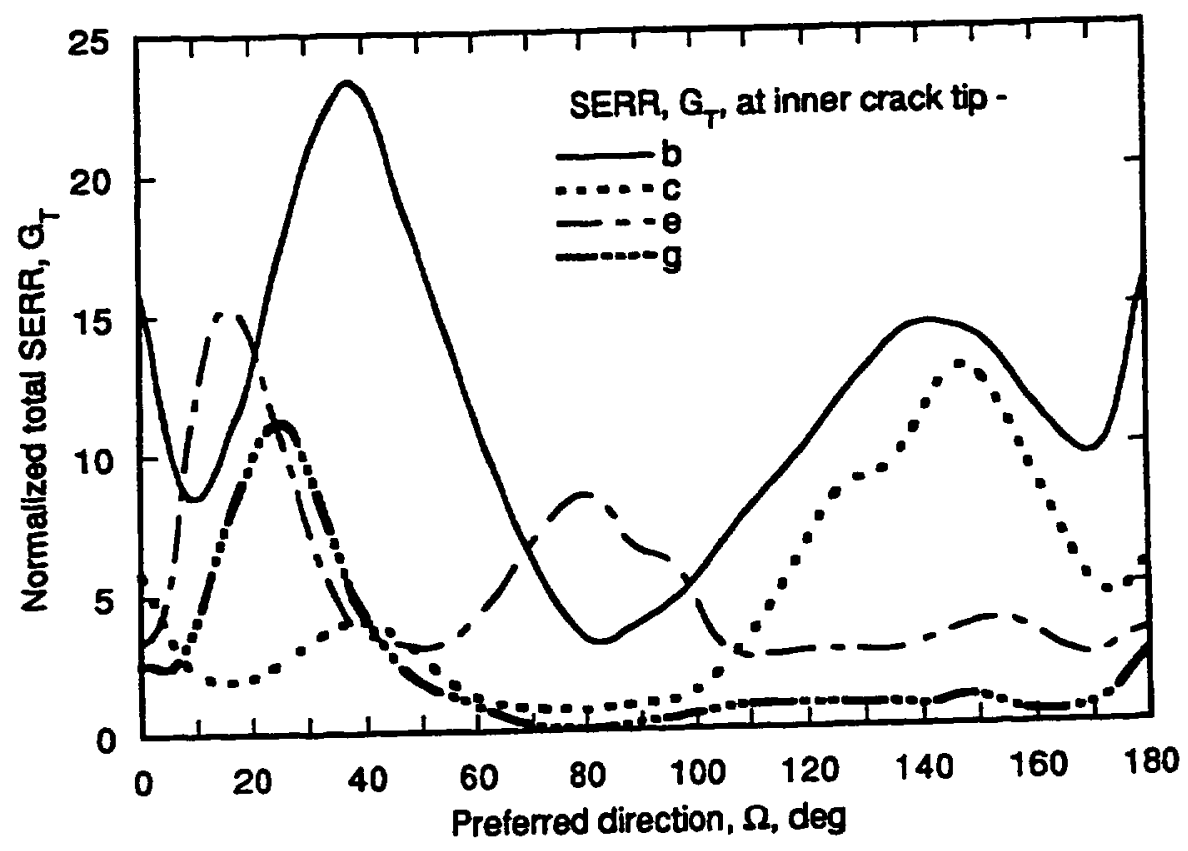

Figure 26: Normalized $G_{T}$ versus $\Omega$ for notch and three microcrack inner tips in a composite plate (strength of anisotropy $E_{L L} / E_{T T}=40$; tip distance, $0.005 a_{1}$; subjected to far-field normal stress, see Fig. 25).

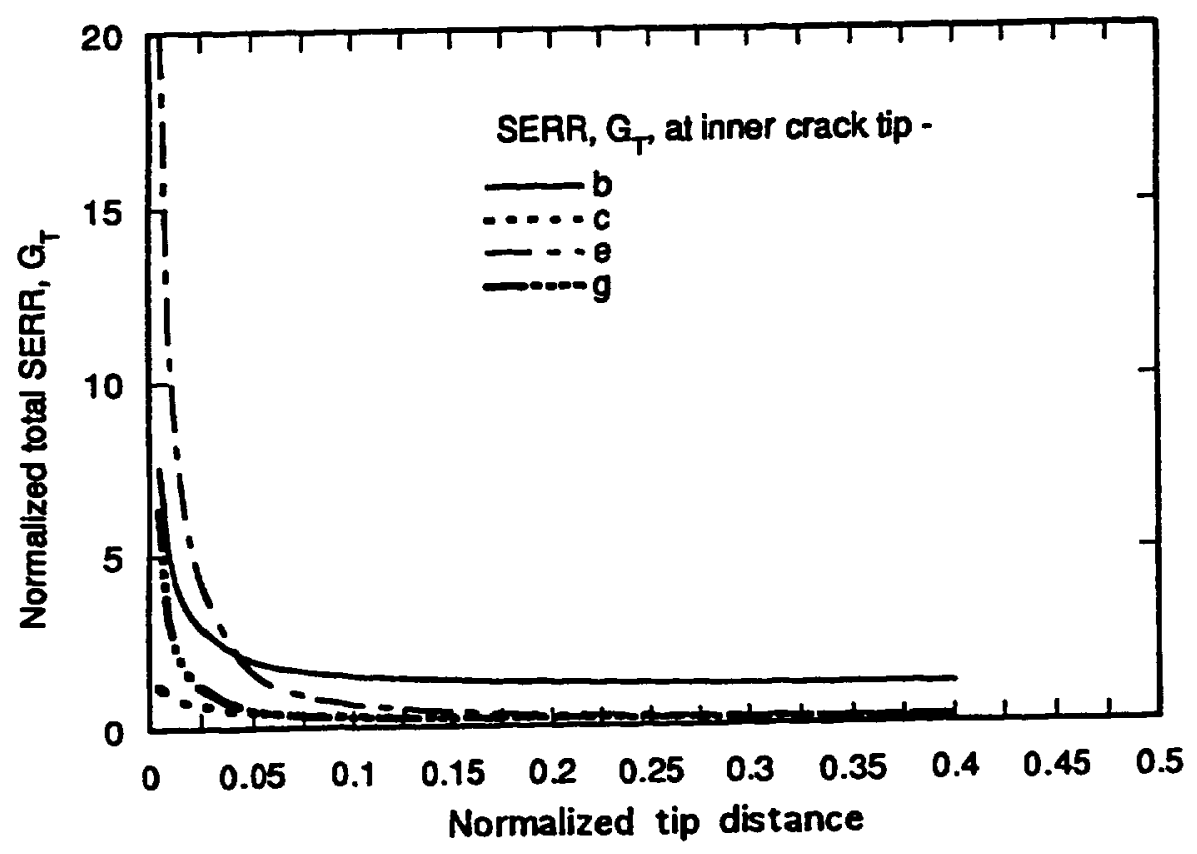

Figure 27: Normalized $\mathrm{G}_{T}$ versus normalized tip distance between inner tips of notch and three microcrack in a composite plate (strength of anisotropy $E_{L L} / E_{T T}=40$; reinforced at $\Omega=15^{\circ}$; subjected to far-field normal stress, see Fig. 25 ). 


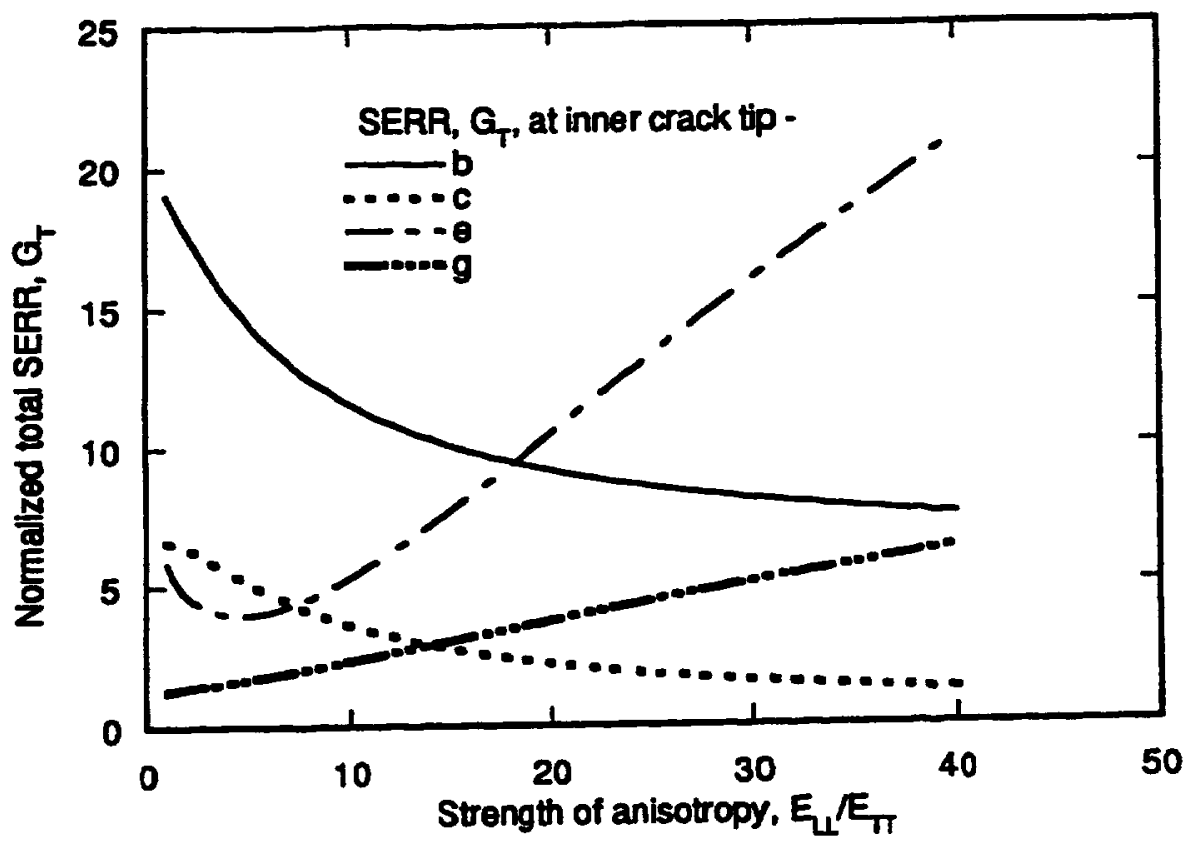

Figure 28: Normalized $G_{T}$ versus strength of anisotropy $E_{L L} / E_{T T}$ for inner tips of notch and three microcracks in a composite plate (reinforced at $\Omega=15^{\circ}$, tip distance, $0.005 a_{1}$; subjected to far-field normal stress, see Fig. 25).

the $G_{T}$ 's of the remaining microcrack inner tips. However, even a small change in the strength of anisotropy significantly influences the value of the $G_{T}$ for all inner crack tips. Note that when $E_{L L} / E_{T T}>18$, the $G_{T}$ for the inclined microcrack tip $e$ becomes the largest. And since the crack having the highest $G_{T}$ will propagate first, a kinked crack will be generated by connection of crack ef to notch $a b$.

\section{CONCLUDING REMARKS}

A rigorous formulation has been presented for calculating the crack-opening displacement, SIF and $G_{T}$ at the various crack tips of a multicracked anisotropic medium. This formulation has been shown to simplify exactly to our previous isotropic formulation which was validated for a number of published crack orientations. The size, orientation, and distribution of all cracks were considered to be independent parameters of the solution. This unique formulations is computationally efficient and offers accurate solution capability. It allows us to easily perform numerous parametric studies to analyze the contribution of each parameter on the local stress field and the characteristics of the damage progression in an anisotropic (e.g., transversely isotropic) material.

The problems of two and three, collinear and non-collinear, interacting cracks were examined. By varying the strengths of anisotropy, we showed that materials with proferred off-axis directions relative to the applied load produced highly mixed-mode crack propagation, even when only mode-I type crack geometry was present. A small change in the strength of anisotropy (when $0<E_{L L} / E_{T T}<5$ ) was shown to highly influence 
the $\mathrm{G}_{T}$ and crack-opening displacements for a given tip. These parameters then reached a plateau when $E_{L L} / E_{T T}>15$. Consequently, even slightly anisotropic materials should be analyzed by using the fully anisotropic approach discussed in this paper.

Modified stiffness parameters (MSP's) were presented as a function of strength of anisotropy and preferred direction. We concluded that $E_{x y}^{(1)}$ and $E_{y y}^{(2)}$ are proportional to the square root of the effective Young's module in the local $x$-and $y$-direction, respectively, and that the other two MSP's are identical (i.e., $E_{z y}^{(2)}=E_{y y}^{(1)}$ ) and related to $b_{16}$ and $b_{26}$ such that they vanish under orthotropic and isotropic conditions.

The discrete auxiliary functions were shown to be related to the crack-opening displacements $\Delta u$ and $\Delta v$. We showed that for cases in which the preferred direction and the applied stress do not coincide (i.e., off-axis orientations), a mode-I local stress field is produced under mixed-mode local deformations, or alternatively, mode-I normal deformation results in a mixed-mode local stress field. Furthermore, the total $G_{T}$ was shown to be the most complete anisotropic fracture parameter (because of its sensitivity in detecting changes in the strength of anisotropy and preferred direction). Thus we suggest that for an anisotropic material it should be used as the crack propagation criterion instead of the SIF.

Interaction effects were demonstrated for all fracture parameters. Amplification of the $G_{T}$ and/or the SIF was shown to occur when cracks were located in front of the main crack. Conversely, reductions in the $G_{T}$ or SIF were observed when shielding of a crack, by other cracks located above and/or below it, was present. Finally, stress or damage channeling was discovered to play a significant role in the mechanisms that govern crack interaction, in that, stresses were channeled along the preferred direction, causing nonsymmetric interaction, even in the presence of symmetric crack configurations.

\section{References}

[1] Timoshenko, S.; Goodier, I.N. (1969):Theory of Elasticity. Third ed., McGraw Hill, New York.

[2] Binienda, W.K.; Arnold, S.M.; Tan, H.Q.; and Xu, M.H. (1993): Stress Intensity Factors in a Fully Interacting, Multicracked, Isotropic Plate. Comp. Mech., Vol. 12, no. 5, pp. 297-314.

[3] Erdogan F. (1978) Mixed Boundary-Value Problems in Mechanics. Mechanics Today, S. Nemat-Nasser ed., Vol. 4, Pergamon Press, New York, pp. 1-32.

[4] Badaliance R.; Gupta,G. G. (1976): Growth Characteristics of Two Interacting Cracks. Eng. Fract. Mech., Vol. 8, no. 2, pp. 341-353.

[5] Delale, F.; Erdogan,F. (1977): The Problem of Internal and Edge Cracks in an Orthotropic Strip., J. Appl. Mech., Vol. 44, no. 6, pp. 237-242.

[6] Delale, F.; Erdogan, F. (1979): Bonded Orthotropic Strips with Cracks. Int. J. Fract., Vol. 15, no. 8, pp. 343-364.

[7] Delale, F.; Bakirtas,I; Erdogan, F. (1979): The Problem of an Inclined Crack in an Orthotropic Strip., J. Appl. Mech., Vol. 46, no.3, pp. 90-96. 
[8] Cherepanov G.P., "Mechanics of Brittle Fracture", McGraw-Hill, N.Y. 1979.

[9] Horii, H.; Nemat-Nasser, S. (1985): Elastic Fields of Interacting Inhomogeneities. Int. J. Solids Struct., Vol. 21, no. 7, pp. 731-745.

[10] Agarwal, B.D., and Broutman, L.J.(1980): Analysis and Performance of Fiber Composites, John Wiley \& Sons, Inc.

[11] Erdogan F. (1962): On The Stress Distribution in Plates with Collinear Cuts Under Arbitrary Loads. Proceedings of the Fourth U.S. National Congress of Applied Mechanics, Vol. 1,ASME, New York, pp. 547-553.

[12] Spencer, A.J.M. (1972): Deformations of Fibre-reinforced Materials, Clarendon Press, Oxford. 

Pubtic reporting burden for this collection of information is estimated to average 1 hour per response, including the lima for reviewing instructions, searching existing data sources, gathoring and malntaining the data needed, and completing and reviewing the collection of intormation. Send comments regarding this burdon estimate or any other aspect of this Davis Highway, Sulte 1204, Aslington, VA 22202-4302, and to the Oftice of Managernent and Budget. Papenwork Reduction Projed (0704-0188), Washington, DC 20503.

\begin{tabular}{l|l|l} 
1. AGENCY USE ONLY (Leave blank) & 2. REPORT DATE & 3. REPORT TYPE AND DATES COVERED
\end{tabular}

\section{TILE AND SUBTITLE} January 1995

Driving Force Analysis in an Infinite Anisotropic Plate With Multiple Crack Interactions

6. AUTHOR(S)

Wieslaw K. Binienda and Steven M. Amold 5. FUNDING NUMBERS

WU-505-63-12

NAG3-1223

8. PERforming organization REPORT NUMBER

E-9401

National Aeronautics and Space Administration

Lewis Research Center

Cleveland, Ohio 44135-3191

9. SPONSORING/MONTORING AGENCY NAME(S) AND ADDRESS(ES)

National Aeronautics and Space Administration

Washington, D.C. 20546-0001

11. SUPPLEMENTARY NOTES

Wieslaw K. Binienda, The University of Akron, Akron, Ohio 44325-3905 (work funded by NASA Grant

NAG3- 1223). Responsible person, Steven M. Amold, organization code 5220, (216) 433-3334.

12a. DISTRIBUTIONAVAILABILTYY STATEMENT

12b. DISTRIBUTION CODE

Unclassified - Unlimited

Subject Category 24

This publication is available from the NASA Center for Aerospace Information, (301) 621-0390.

13. ABSTRACT (Maximum 200 words)

The methodology and a rigorous solution formulation are presented for stress intensity factors (SIFs, $k$ ) and total strain energy release rates (SERR, $\mathrm{G}_{T}$ ) of a multicracked plate, that has fully interacting cracks and is subjected to a far-field arbitrary stress state. The fundamental perturbation problem is derived, and the steps needed to formulate the system of singular integral equations whose solution gives rise to the evaluation of the SIF's are identified. Parametric studies are conducted for two, three and four crack problems. The sensitivity and characteristics of the model is demonstrated.

14. SUBJECT TERMS

Fracture mechanics; Anisotropy; Multiple cracks; Elastic

OF REPORT

Unclassified
18. SECURITY CLASSIFICATION

OF THIS PAGE Unclassified
19. SECURITY CLASSIFICATION

OF ABSTRACT

Unclassified
15. NUMBER OF PAGES

16. PRICE COOE

A03

20. LIMITATION OF ABSTRACT

20. LIMITATION OF ABSTRACT

Standard Form 298 (Rev. 2-89)

Prescribed by ANSI Std. Z39-18

298.102 